\title{
Statistische Krankenhausdaten: Grunddaten der Krankenhäuser 2017
}

\begin{abstract}
Elektronisches Zusatzmaterial Die elektronische Version dieses Kapitels enthält Zusatzmaterial, das den Nutzern zur Verfügung steht unter https://doi.org/10.1007/978-3-662-58225-1_17.
\end{abstract}

\section{Ute Bölt}

(c) Der/die Autor(en) 2019

J. Klauber et al. (Hrsg.), Krankenhaus-Report 2019

https://doi.org/10.1007/978-3-662-58225-1_17

\section{Zusammenfassung}

Dieser Beitrag fasst die Ergebnisse der Krankenhausstatistik zu den Grunddaten der Krankenhäuser für das Berichtsjahr 2017 zusammen. Er gibt einen Überblick über die sachlichen und personellen Ressourcen ( $z$. B. Betten, Fachabteilungen, Personal) sowie die Inanspruchnahme von Krankenhausleistungen (Patientenbewegungen). Die Krankenhausstatistik ist eine seit 1991 bundeseinheitlich durchgeführte jährliche Vollerhebung. Auskunftspflichtig sind die Träger der Krankenhäuser. Die Diagnosedaten der Krankenhauspatienten werden wie die fallpauschalenbezogene Krankenhausstatistik (DRG-Statistik) jeweils in einem gesonderten Beitrag behandelt ( $\triangleright$ Kapitel 18 und 19).

The article summarises the results of the hospital statistics for the reporting year 2017. It provides an overview of the material and personnel resources in German hospitals, (e.g. beds, specialist departments, staff) and the utilisation of hospital services (patient movements). The survey has been carried out annually on a nationwide basis since 1991. Hospital owners are obliged to disclose information. The DRG statistics as well as the inpatient diagnosis statistics are described in separate articles ( $\$$ chapter 18 and 19).

\subsection{Vorbemerkung}

Die Krankenhausstatistik des Statistischen Bundesamtes liefert vielfältige Informationen über das Volumen und die Struktur des Leistungsangebots sowie über die Inanspruchnahme von Krankenhausleistungen. Seit 1991 umfasst die jährlich durchgeführte Vollerhebung die Krankenhäuser im gesamten Bundesgebiet. Das Erhebungsprogramm gliedert sich in die Grunddaten der Krankenhäuser, den Kostennachweis der Krankenhäuser und die Diagnosen der Krankenhauspatienten. ${ }^{1}$ Die fallpauschalenbezogene Krankenhausstatistik (DRG-Sta-
1 Eine ausführliche Darstellung der Ergebnisse der Krankenhausstatistik enthält die Fachserie 12 (Gesundheit) des Statistischen Bundesamtes. Die jährlich publizierten Reihen 6.1.1 (Grunddaten der Krankenhäuser) und 6.3 (Kostennachweis der Krankenhäuser) sind auf der Themenseite Gesundheit des Statistischen Bundesamtes unter Veröffentlichungen im Bereich Krankenhäuser (in der Regel kostenfrei) erhältlich. Die Reihen 6.2.1 (Diagnosen der Krankenhauspatienten) und 6.4 (Fallpauschalenbezogene Krankenhausstatistik - DRG-Statistik) wurden letztmals für das Berichtsjahr 2016 veröffentlicht. Aktuelle Ergebnisse zu den Diagnosedaten der Patienten und Patientinnen in Krankenhäusern (Code 23131) und zur Fallpauschalenbezogenen Krankenhausstatistik (Code 23141) stehen in der Datenbank GENESIS-Online - auch als lange Reihen - zur Verfügung. Weitere Informationen können unter gesundheit@destatis.de angefordert werden. 
tistik - Diagnosis Related Groups Statistics) ergänzt seit 2005 die Krankenhausdiagnosestatistik insbesondere um Angaben zu Operationen und medizinischen Prozeduren bei stationären Patienten. Gegenstand der folgenden Betrachtung sind die Grunddaten der Krankenhäuser. Eine ausführliche Darstellung der Krankenhausdiagnosestatistik enthält $>$ Kapitel 18, Ergebnisse der DRG-Statistik werden in $>$ Kapitel 19 präsentiert.

Rechtsgrundlage ist die 1990 in Kraft getretene und im Jahr 2001 erstmals umfassend novellierte Krankenhausstatistik-Verordnung (KHStatV). Die Novellierung war erforderlich geworden, um die Krankenhausstatistik an die Entwicklungen im Bereich der stationären Gesundheitsversorgung anzupassen. ${ }^{2}$ Weitere wesentliche Änderungen gibt es ab 2007 bei der Erhebung der Kosten der Ausbildungsstätten (Wegfall der Ausbildungsstätten-Umlage) und der neu hinzugekommenen gesonderten Erfassung von Aufwendungen für den Ausbildungsfonds ${ }^{3}$ sowie ab 2009 bei der zusätzlichen Erhebung von Personal ohne direktes Beschäftigungsverhältnis beim Krankenhaus und die hierauf entfallenden Sachkosten. ${ }^{4}$ Der vorliegende Beitrag schließt sich an das Kapitel 19 im Krankenhaus-Report 2018 an. Die Struktur des Kapitels orientiert sich am Angebot und der Inanspruchnahme von Krankenhausleistungen. An einen ersten Überblick über die Ergebnisse des Jahres 2017 anhand ausgewählter Kennzahlen der Krankenhäuser $(\checkmark$ Abschn. 17.2) schließt sich eine detaillierte Betrachtung des Angebots von Krankenhausleistungen an $(\checkmark$ Abschn. 17.3). Dabei wird auf die sachliche, personelle und fachlich-medizinische Ausstattung der Krankenhäuser eingegangen. Im Weiteren werden Ergebnisse zur Inanspruchnahme von Krankenhausleistungen nach unterschiedlichen Behandlungsformen präsentiert ( $\triangleright$ Abschn. 17.4).

$2 \mathrm{Zu}$ inhaltlichen und methodischen Änderungen aufgrund der ersten Novellierung der Krankenhausstatistik-Verordnung siehe Rolland S, Rosenow C (2005) Statistische Krankenhausdaten: Grund- und Kostendaten der Krankenhäuser 2002. In: Klauber J, Robra BP, Schellschmidt H (Hrsg) Krankenhaus-Report 2004. Schattauer, Stuttgart, S. 291-310.

3 Aufwendungen nach $\S 17$ a Krankenhausfinanzierungsgesetz (KHG) zur Finanzierung von Ausbildungsstätten und -vergütungen.

4 Art. 4b des Krankenhausfinanzierungsreformgesetzes vom 24. März 2009.

\subsection{Kennzahlen der Krankenhäuser}

Im Hinblick auf den Beitrag „Fallpauschalenbezogene Krankenhausstatistik: Diagnosen und Prozeduren der Krankenhauspatienten auf Basis der Daten nach $\$ 21$ Krankenhausentgeltgesetz “ $\left(\triangleright\right.$ Kapitel $\left.19^{5}\right)$, der sich ausschließlich mit dem Behandlungsgeschehen in allgemeinen Krankenhäusern befasst, werden vorab die Besonderheiten allgemeiner Krankenhäuser im Vergleich zu sonstigen Krankenhäusern anhand ausgewählter Kennzahlen dargestellt. Alle weiteren Ausführungen in diesem Kapitel zu den „Statistische(n) Krankenhausdaten: Grunddaten der Krankenhäuser 2017“ beziehen sich auf die Gesamtheit der Krankenhäuser in Deutschland.

\subsubsection{Allgemeine und sonstige Krankenhäuser im Vergleich}

Von 1.942 Krankenhäusern insgesamt sind 1.592 allgemeine und 285 sonstige Krankenhäuser (ohne 65 reine Tages- und Nachtkliniken mit ausschließlich teilstationärer Versorgung). Allgemeine Krankenhäuser sind Einrichtungen mit einem in der Regel breiten Behandlungsspektrum. Sie verfügen deshalb über ein entsprechendes Angebot verschiedener Fachabteilungen. Davon zu unterscheiden sind Krankenhäuser, deren Schwerpunkt im psychiatrischen Bereich liegen. Da neben einem Angebot an psychiatrischen Fachabteilungen in diesen Einrichtungen oft auch noch neurologische oder geriatrische Behandlungsschwerpunkte kombiniert werden, versteht man unter den sonstigen Krankenhäusern Einrichtungen mit ausschließlich psychiatrischen und psychotherapeutischen Betten, mit psychiatrischen, psychotherapeutischen und neurologischen Betten, mit psychiatrischen, psychotherapeutischen und geriatrischen Betten sowie mit

5 Krankenhäuser, die nach dem DRG-Vergütungssystem abrechnen und dem Anwendungsbereich des $\S 1$ KHEntgG unterliegen (hier: allgemeine Krankenhäuser), bilden die Datenbasis für die DRG-Statistik. Die Anwendung eines pauschalierenden Entgeltsystems auch für psychiatrische und psychosomatische Einrichtungen (hier: sonstige Krankenhäuser ohne reine Tages- und Nachtkliniken) ist nach $\S 17$ d Abs. 1 KHG ab 1. Januar 2018 vorgesehen. 
Tab. 17.1 Eckdaten verschiedener Krankenhaustypen: Allgemeine Krankenhäuser und Sonstige Krankenhäuser (OHNE reine Tages- und Nachtkliniken) im Vergleich

\begin{tabular}{|c|c|c|c|c|c|c|}
\hline \multicolumn{4}{|c|}{ Gegenstand der Nachweisung } & $\begin{array}{l}\text { Krankenhäuser } \\
\text { insgesamt }\end{array}$ & $\begin{array}{l}\text { Allgemeine } \\
\text { Krankenhäuser }\end{array}$ & $\begin{array}{l}\text { Sonstige } \\
\text { Krankenhäuser }^{*}\end{array}$ \\
\hline \multicolumn{4}{|c|}{ Anzahl der Krankenhäuser } & 1.942 & 1.592 & 285 \\
\hline \multicolumn{7}{|c|}{ Krankenhäuser mit ... Betten } \\
\hline \multicolumn{4}{|c|}{ unter 100} & 666 & 477 & 124 \\
\hline \multicolumn{4}{|c|}{$100-199$} & 439 & 363 & 76 \\
\hline \multicolumn{4}{|c|}{ 200-499 } & 557 & 481 & 76 \\
\hline \multicolumn{4}{|c|}{500 und mehr } & 280 & 271 & 9 \\
\hline \multicolumn{4}{|c|}{ Aufgestellte Betten } & 497.182 & 450.453 & 46.729 \\
\hline \multicolumn{4}{|c|}{ Bettenauslastung } & 77,8 & 76,2 & 92,6 \\
\hline \multicolumn{4}{|c|}{ Stationär beh. Patienten } & 19.442 .810 & 18.824 .723 & 618.087 \\
\hline \multicolumn{4}{|c|}{ Berechnungs-/Belegungstage } & 141.151 .861 & 125.351 .056 & 15.800 .805 \\
\hline \multicolumn{4}{|c|}{ Durchschn. Verweildauer in Tagen } & 7,3 & 6,7 & 25,6 \\
\hline \multicolumn{4}{|c|}{ Vollkräfte im Jahresdurchschnitt } & 894.400 & 831.009 & 62.508 \\
\hline \multirow[t]{7}{*}{ davon: } & \multicolumn{3}{|c|}{ Ärztliches Personal } & 161.208 & 153.861 & 7.201 \\
\hline & \multicolumn{3}{|c|}{ Nichtärztliches Personal } & 733.193 & 677.148 & 55.307 \\
\hline & \multirow[t]{5}{*}{ davon: } & \multicolumn{2}{|c|}{ Pflegedienst } & 328.327 & 297.292 & 30.805 \\
\hline & & dar.: & $\begin{array}{l}\text { In der Psychiatrie } \\
\text { tätig }\end{array}$ & 46.275 & 18.187 & 27.869 \\
\hline & & Med. & ch. Dienst & 149.655 & 139.152 & 10.252 \\
\hline & & Funk & nsdienst & 109.199 & 105.911 & 3.170 \\
\hline & & Übrig & Personal & 146.012 & 134.794 & 11.081 \\
\hline \multicolumn{7}{|c|}{$\begin{array}{l}\text { *) Zu den Sonstigen Krankenhäusern rechnen (neben reinen Tages- und Nachtkliniken) Krankenhäuser mit } \\
\text { - ausschließlich psychiatrischen und psychotherapeutischen Betten } \\
\text { - psychiatrischen, psychotherapeutischen und neurologischen Betten } \\
\text { - psychiatrischen, psychotherapeutischen und geriatrischen Betten } \\
\text { - psychiatrischen, psychotherapeutischen, neurologischen und geriatrischen Betten }\end{array}$} \\
\hline
\end{tabular}

psychiatrischen, psychotherapeutischen, neurologischen und geriatrischen Betten (•Tab. 17.1).

Der Anteil kleinerer Häuser mit weniger als 100 Betten liegt bei den sonstigen Krankenhäusern bei 43,5 \% (30,0 \% bei allgemeinen Krankenhäusern), lediglich 3,2 \% der Häuser verfügen über 500 und mehr Betten (17,0 \% bei allgemeinen Krankenhäusern). Von 19,4 Millionen stationär behandelten Patientinnen und Patienten wurden zwar nur 3,2 \% in einem sonstigen Krankenhaus behandelt; allerdings entfielen auf diese Patientinnen und Patienten
$11,2 \%$ der insgesamt gut 141 Millionen Berechnungs- und Belegungstage des Jahres 2017. Daraus errechnet sich eine durchschnittliche Verweildauer von 25,6 Tagen, die sich aus dem besonderen Behandlungsspektrum dieser Einrichtungen ergibt. Überwiegend werden dort psychische Erkrankungen behandelt. Demgegenüber dauerte der Aufenthalt für die Patientinnen und Patienten in allgemeinen Krankenhäusern lediglich 6,7 Tage. Die lange Verweildauer wirkt sich positiv auf die Bettenauslastung in sonstigen Krankenhäusern aus. Sie liegt 
mit 92,6 \% um 16,4 Prozentpunkte über der Bettenauslastung allgemeiner Krankenhäuser (76,2 \%).

In sonstigen Krankenhäusern sind lediglich $11,5 \%$ der beschäftigten Vollkräfte dem ärztlichen Personal zuzurechnen, in allgemeinen Krankenhäusern sind 18,5 \% der Vollkräfte Ärzte. Mehr als die Hälfte der Vollkräfte im nichtärztlichen Dienst $(55,7 \%)$ gehört in den sonstigen Krankenhäusern zum Pflegedienst, in allgemeinen Krankenhäusern liegt der Anteil der Pflegevollkräfte an den nichtärztlichen Vollkräften bei 43,9\%.

Alle weiteren Ausführungen in diesem Kapitel zu den Statistischen Krankenhausdaten: Grunddaten der Krankenhäuser 2017 beziehen sich auf die Gesamtheit der Krankenhäuser in Deutschland.

\subsubsection{Krankenhäuser insgesamt}

Einen Überblick über zentrale Ergebnisse des Jahres 2017, auf die in den folgenden Abschnitten intensiver eingegangen wird, gibt $\bullet$ Tab. 17.2. Die kompletten Ergebnisse für die Jahre 1991 bis 2017 finden sich als Zusatzmaterial auf SpringerLink unter https://doi.org/10.1007/978-3-662-58225-1 (Zusatztabellen 17.a und 17.b). Zu den grundlegenden Kennzahlen von Krankenhausleistungen gehören auf der
Angebotsseite die Anzahl der Einrichtungen, Betten und Beschäftigten. Unter dem Gesichtspunkt der Inanspruchnahme stellen die Anzahl der vollstationären Krankenhausfälle und die durchschnittliche Verweildauer wesentliche Kennzahlen dar.

Um einen Eindruck von der kurz-, mittel- und langfristigen Entwicklung der einzelnen Indikatoren zu gewinnen, wird der Überblick um einen Vorjahres-, 5- und 10-Jahres-Vergleich erweitert. Ergänzend stellt $\bullet$ Abb. 17.1 die zeitliche Entwicklung der wesentlichen Kennzahlen grafisch dar.

\subsection{Die Ressourcen der Krankenhäuser}

Das Angebot der Krankenhäuser setzt sich aus einer sachlichen, einer personellen und einer fachlichmedizinischen Komponente zusammen. Die sachliche Ausstattung wird neben der Einrichtungszahl vor allem durch die Anzahl der aufgestellten Betten sowie der medizinisch-technischen Großgeräte ( $\downarrow$ Abschn. 17.3.1) bestimmt. Das fachlich-medizinische Angebot der Krankenhäuser spiegelt sich in den Fachabteilungen wider ( $\triangleright$ Abschn. 17.3.2). Aussagen über die Verteilung der Ressourcen nach Disziplinen sind auf Basis der Bettenzahl nach Fachab-

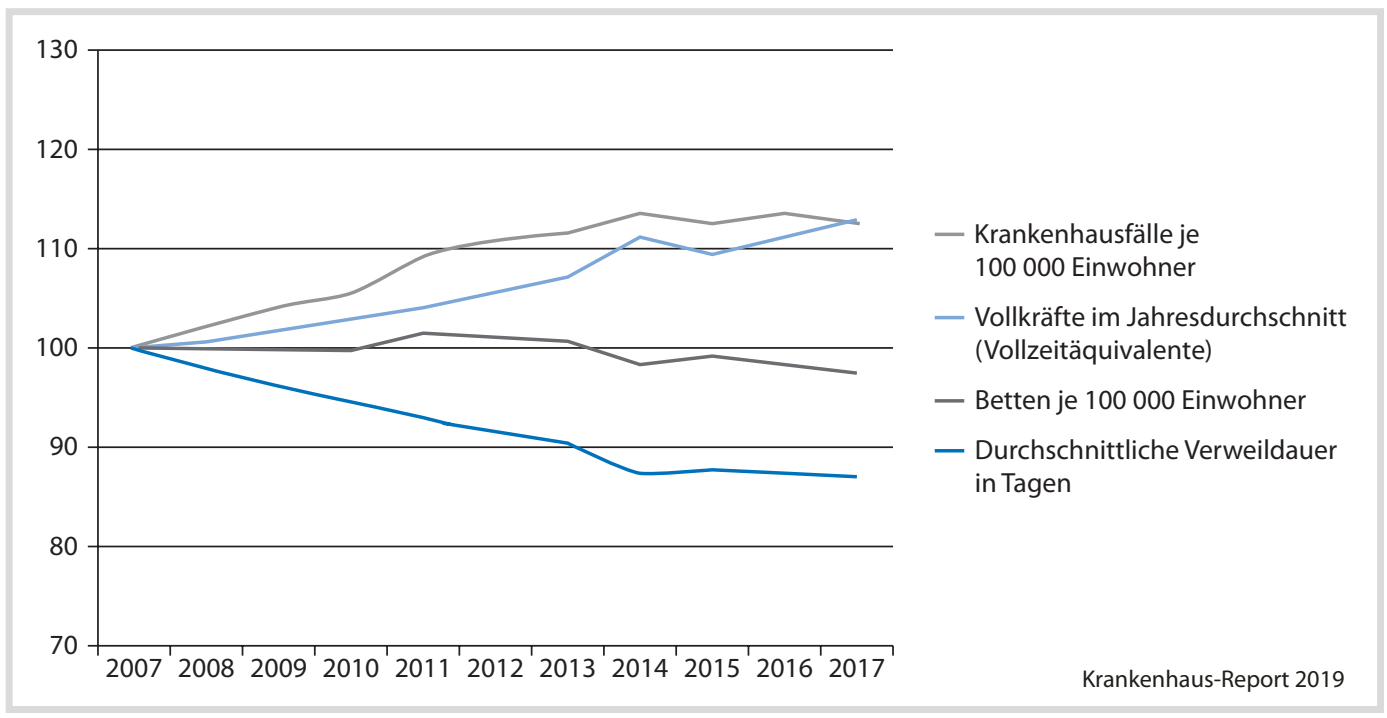

- Abb. 17.1 Entwicklung zentraler Indikatoren der Krankenhäuser 2007 bis 2017 (Index 2007 = 100) (Quelle: Statistisches Bundesamt (Destatis) 2018, Grunddaten der Krankenhäuser) 


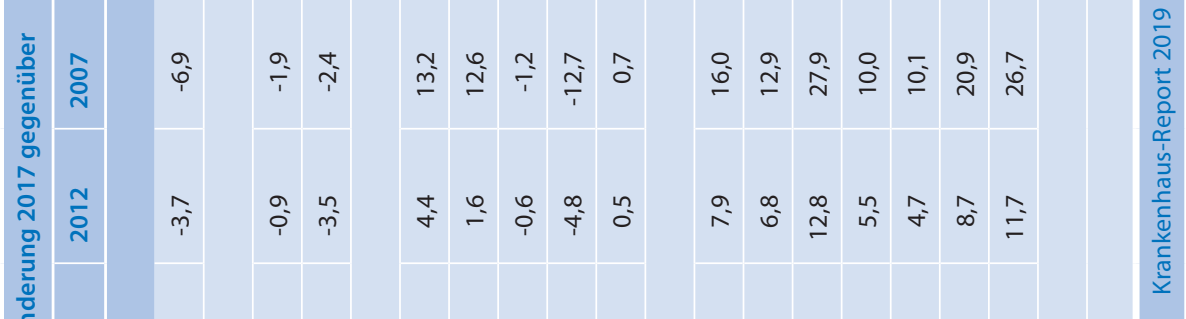

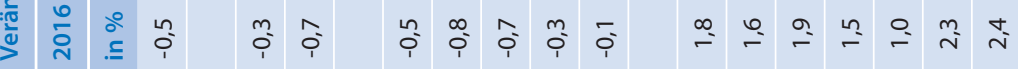

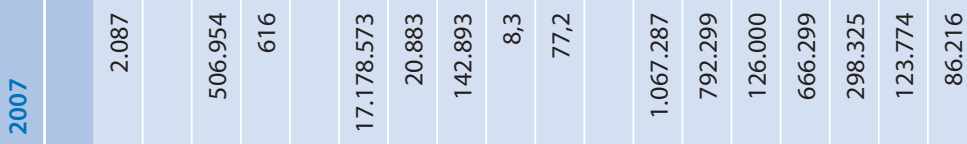

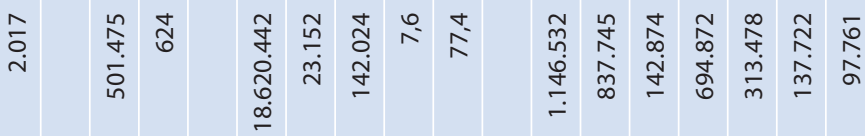

$\frac{\text { ก }}{2}$

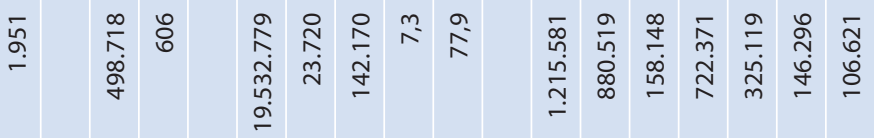

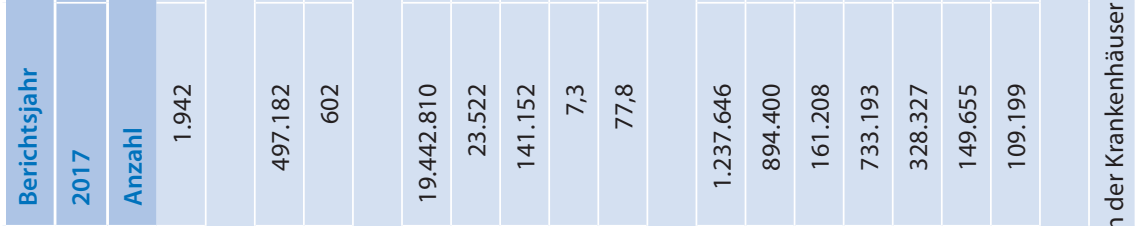

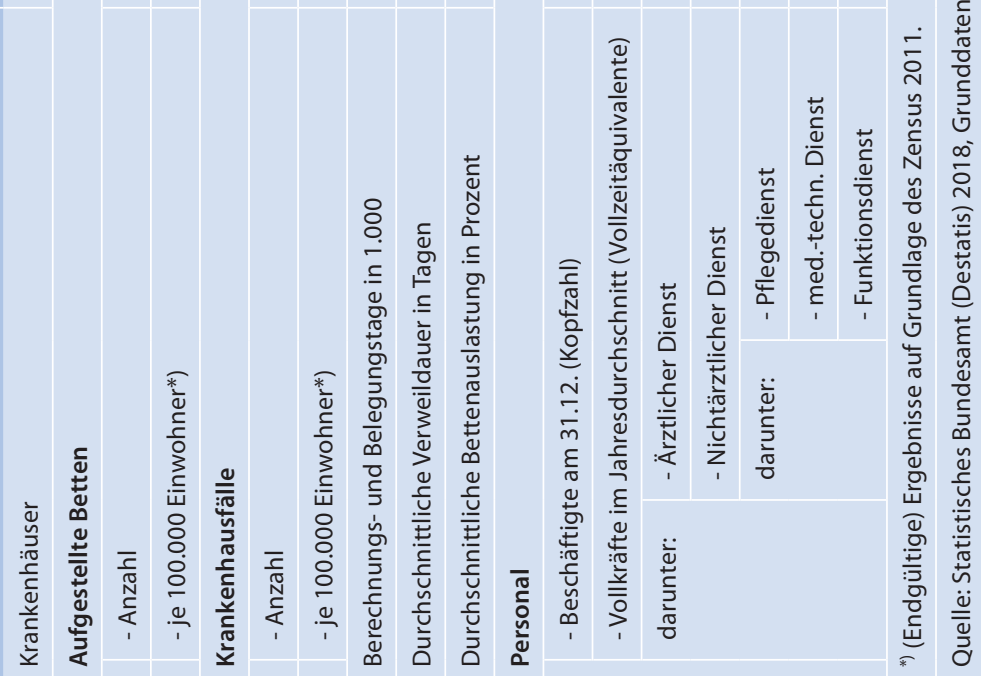




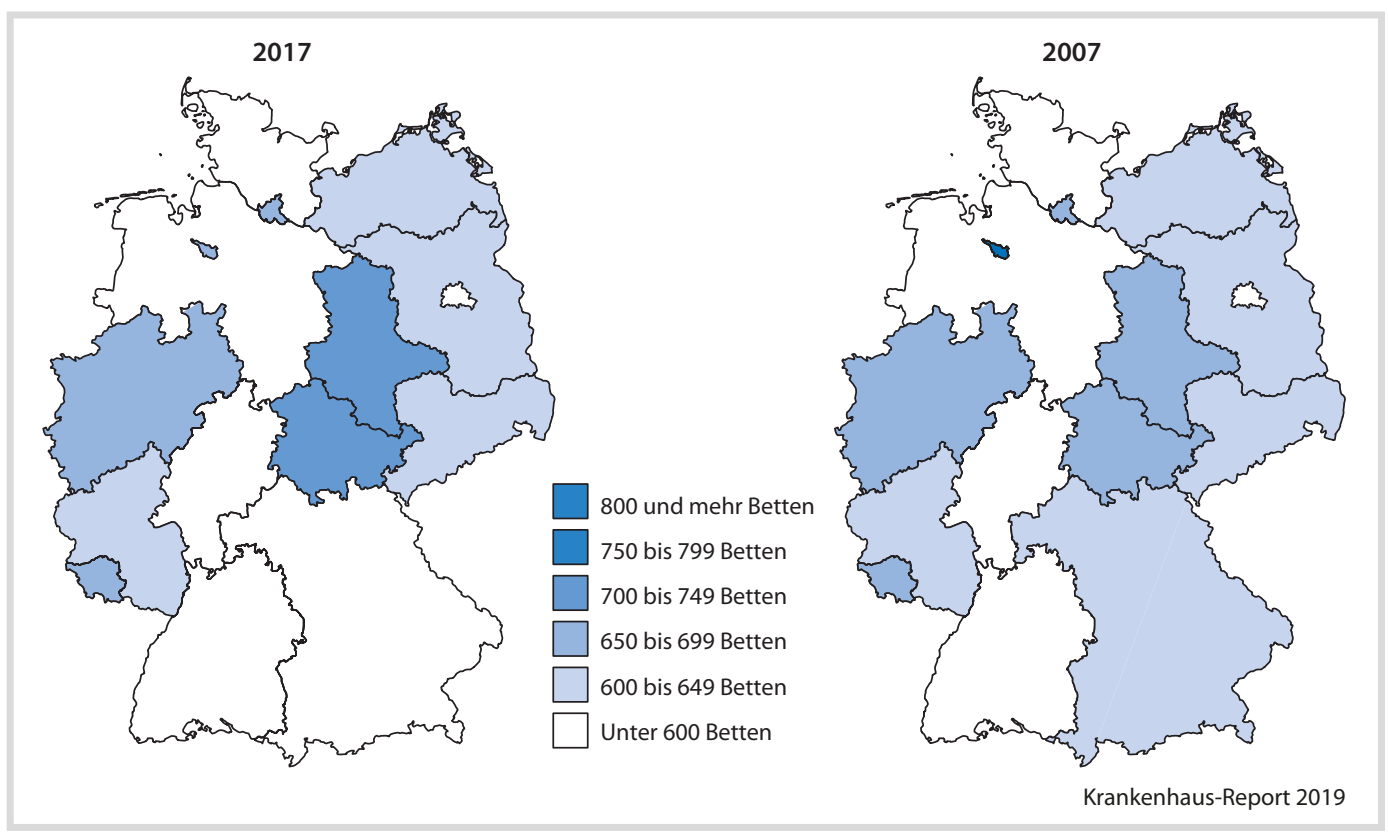

- Abb. 17.2 Bettendichte im Ländervergleich 2007 und 2017 (Quelle: Statistisches Bundesamt (Destatis) 2018, Grunddaten der Krankenhäuser)

teilungen möglich. Besondere Bedeutung kommt im dienstleistungsorientierten Krankenhausbetrieb der personellen Ausstattung der Krankenhäuser mit ärztlichem und pflegerischem Personal zu. Darüber hinaus stellen Krankenhäuser wichtige Arbeitgeber im Gesundheitswesen dar und fungieren als Ausbildungsstätten für Gesundheitsberufe ( $\triangleright$ Abschn. 17.3.3).

\subsubsection{Sachliche Ausstattung}

Eine bedarfsgerechte Versorgung der Bevölkerung sicherzustellen ist das Ziel der Krankenhausplanung ${ }^{6}$, die in zahlreichen Bundesländern auf der in

6 Krankenhausplanung der Länder gem. $§ 6$ des Gesetzes zur wirtschaftlichen Sicherung der Krankenhäuser und zur Regelung der Krankenhauspflegesätze - Krankenhausfinanzierungsgesetz (KHG). Vgl. hierzu zum Beispiel: Dreiundvierzigste Fortschreibung des Krankenhausplans des Freistaates Bayern, Stand 1. Januar 2018, Quelle: Bayerisches Staatsministerium für Gesundheit und Pflege, Internet: www.stmgp.bayern.de/wp-content/uploads/2018/02/ krankenhausplan-des-freistaates-bayern_barrierefrei.pdf den 1960er Jahren in den USA entwickelten HillBurton-Formel ${ }^{7}$ basiert. Im Jahr 2017 standen in insgesamt 1.942 Krankenhäusern Deutschlands 497.182 Betten für die stationäre Gesundheitsversorgung der Bevölkerung zur Verfügung; das Versorgungsangebot blieb gegenüber dem Vorjahr nahezu unverändert (2016: 1.951 Krankenhäuser mit 498.718 Betten). Gegenüber 2007 ging die Zahl der Krankenhäuser infolge von Schließungen, aber auch durch die Fusion ${ }^{8}$ mehrerer ehemals eigenständiger Einrichtungen zu einem Krankenhaus um 145 (6,9\%) zurück. Die Zahl der Krankenhausbetten sank von 506.954 im Jahr 2007 um 9.772 oder $1,9 \%$. Sinkende Bettenzahlen hatten zur Folge, dass sich auch die Bettendichte je 100.000

7 Für die Ermittlung des Bettenbedarfs sind nach der HillBurton-Formel neben der Einwohnerzahl (E) die Krankenhaushäufigkeit (KH), die Verweildauer (VD) und die Bettennutzung (BN) von Bedeutung

Bettenbedarf $=(E \times K H \times V D \times 100) /(1.000 \times[$ Tage im Jahr $] \times B N)$ 8 Zusammenschlüsse zwischen Unternehmen unterliegen unter bestimmten Voraussetzungen der Fusionskontrolle durch das Bundeskartellamt, Internet: http://www.bundeskartellamt.de/DE/Fusionskontrolle. 
Einwohner ${ }^{9}$ verringerte. Bezogen auf die Bevölkerung Deutschlands standen 2017 durchschnittlich 602 Krankenhausbetten je 100.000 Einwohner zur Verfügung; das sind 14 Betten (2,4 \%) weniger als zehn Jahre zuvor.

Die Krankenhausdichte lag bei 2,3 Krankenhäusern je 100.000 Einwohner (2007: 2,5 Krankenhäuser je 100.000 Einwohner).

Knapp ein Fünftel (17,7 \%) aller Krankenhäuser Deutschlands hatte seinen Sitz in Nordrhein-Westfalen; außerdem verfügte das bevölkerungsreichste Bundesland über annähernd ein Viertel (23,8 \%) aller Krankenhausbetten. Die meisten Betten je 100.000 Einwohner gab es jedoch in Bremen (738 Betten), gefolgt von Thüringen (732 Betten) und Sachsen-Anhalt (707 Betten) (• Tab. 17.3). • Abb. 17.2 verdeutlicht die regionalen Unterschiede und die Veränderung der Bettendichte im Vergleich zu 2007. Den stärksten Rückgang verzeichnete Bremen mit einer um 9,9\% niedrigeren Bettendichte gegenüber 2007, gefolgt von Baden-Württemberg mit einem um 9,0\% geringeren Bettenangebot. Die deutlichste Zunahme der Bettendichte gab es hingegen in Thüringen mit einem Plus von 5,3\%, gefolgt von Mecklenburg-Vorpommern mit $+4,6 \%$.

Die Mitversorgungsfunktion, die die Krankenhäuser Bremens für das angrenzende Niedersachsen haben, wird nicht nur durch die Bettendichte, sondern auch durch die weit über dem Bundesdurchschnitt (23.522 Fälle je 100.000 Einwohner) liegende Anzahl der Krankenhausfälle (30.877 je 100.000 Einwohner) deutlich. Aussagen über die Mitversorgungsfunktion einzelner Bundesländer können darüber hinaus anhand der Versorgungsquote $^{10}$ getroffen werden (• Tab. 17.4). Werte über

9 Angaben je 100.000 Einwohner (Betten und Fälle) in den Krankenhausgrunddaten sind ab dem Berichtsjahr 2011 mit der Durchschnittsbevölkerung auf Grundlage des Zensus 2011 ermittelt; bis 2010 basieren die Angaben auf den Durchschnittsbevölkerungen früherer Zählungen.

10 Die Versorgungsquote in der Krankenhausstatistik wird auf Basis der durchschnittlichen Anzahl vollstationär belegter Betten pro Tag ermittelt. Weil für jeden vollstationären Patienten pro Tag, den er in der Einrichtung verbringt, ein Bett belegt wird, kann ein Tag mit einem belegten Bett gleichgesetzt werden. Die Summe der Berechnungs- und Belegungstage wird - jeweils für Wohn- und Behandlungsort - durch die Anzahl der Kalendertage im Berichtsjahr dividiert. Aus der Relation zwischen den belegten Betten nach Wohn- und Behandlungsort ergibt sich die Versorgungsquote.
$100 \%$ besagen, dass die Krankenhäuser eines Bundeslandes mehr Patienten behandelten, als Patienten des jeweiligen Bundeslandes in vollstationärer Behandlung waren. Dies ist insbesondere bei den Stadtstaaten der Fall. So verfügten die Krankenhäuser Bremens 2017 mit 137,2 \% über die höchste Versorgungsquote, gefolgt von Hamburg (136,5\%) und Berlin (110,7). Entsprechend niedrige Versorgungsquoten wiesen die Krankenhäuser der angrenzenden Flächenstaaten auf (Niedersachsen und Schleswig-Holstein: 93,2 \% und 92,5\%, Brandenburg: $88,5 \%)$.

Ergänzend zur Einzugsgebietsstatistik lässt sich der Anteil der Patienten ermitteln, die sich im eigenen Land behandeln ließen. Die Patienten aus Bayern und Nordrhein-Westfalen bevorzugten zu 96,4 \% bzw. 96,3 \% eine vollstationäre Krankenhausbehandlung im eigenen Land. Demgegenüber ließen sich nur 80,0\% der Brandenburger und $82,3 \%$ der Schleswig-Holsteiner im jeweils eigenen Bundesland behandeln.

Die anhand der Anzahl der aufgestellten Betten bestimmte Krankenhausgröße ist ein weiteres Kriterium zur Beurteilung der Strukturen in der Krankenhauslandschaft. Im Jahr 2017 verfügte ein Krankenhaus über durchschnittlich 256 Betten; das sind dreizehn Betten mehr als die durchschnittliche Krankenhausgröße zehn Jahre zuvor (243 Betten).

Der allgemeine Rückgang der Zahl der Krankenhäuser trifft nicht alle Krankenhaustypen gleichermaßen. Die Anzahl sehr kleiner Krankenhäuser mit weniger als 50 Betten (einschließlich reiner Tages- und Nachtkliniken ohne aufgestellte Betten) stieg sogar von 407 im Jahr 2007 auf 430 im Jahr 2017. Das entspricht einer Zunahme des Anteils von 19,5\% im Jahr 2007 um 2,6 Prozentpunkte auf 22,1 \% im Jahr 2017. Mit durchschnittlich 20 Betten verfügte ein Krankenhaus in der Größenklasse 1 bis 49 Betten über zwei Betten weniger als im Jahr 2007. Der Anteil sehr großer Krankenhäuser (800 und mehr Betten) lag 2017 bei 5,0 \%; das sind 0,9 Prozentpunkte mehr als zehn Jahre zuvor (4,1\%); die Durchschnittsgröße dieser Krankenhäuser lag bei 1.217 Betten (2007: 1.199). Trotz des geringen Anteils dieses Krankenhaustyps an den Krankenhäusern insgesamt stand in den sehr großen Krankenhäusern knapp ein Viertel (23,7 \%) aller Betten, in den sehr kleinen Krankenhäusern jedoch nur 1,5 \% 


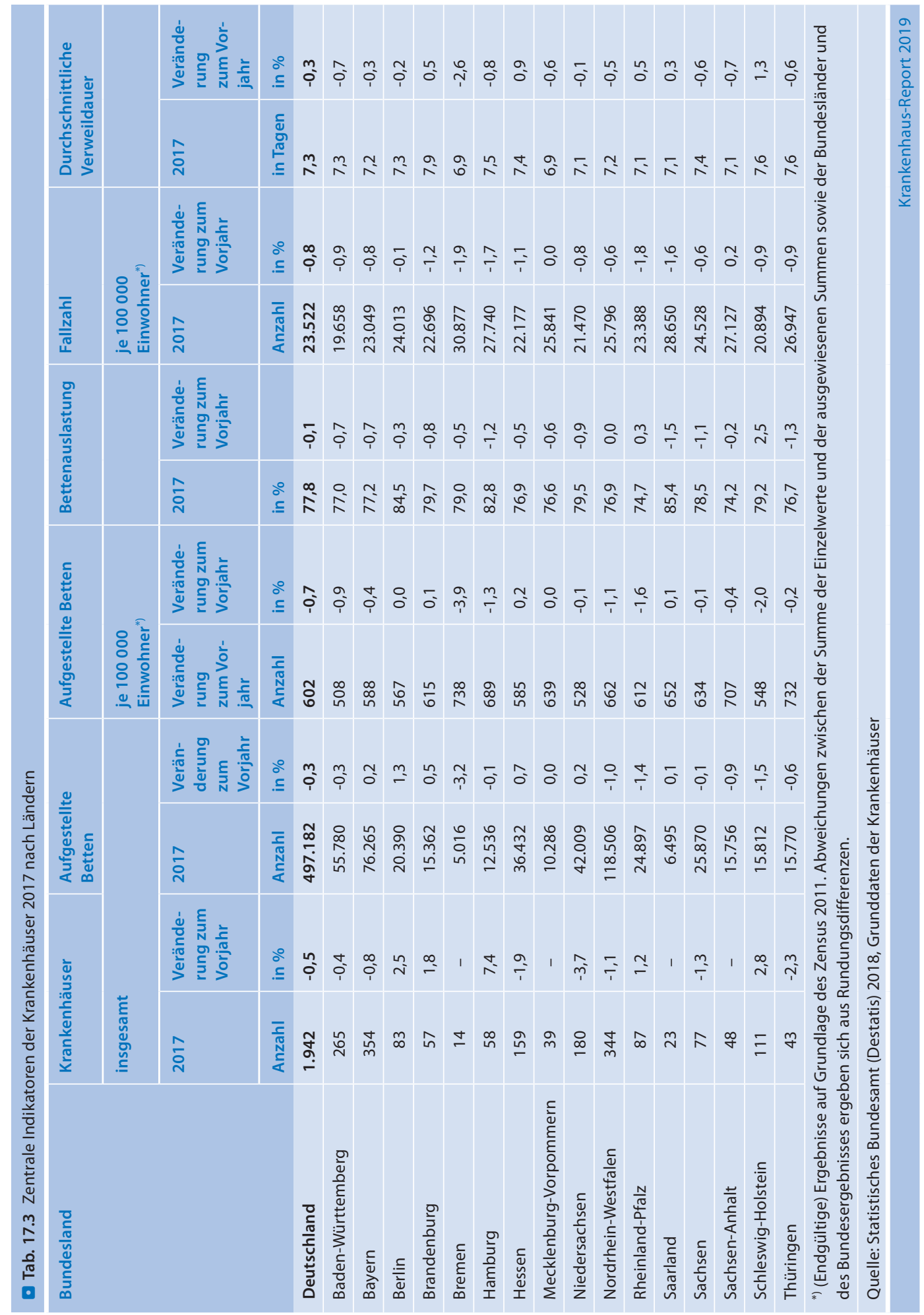


Tab. 17.4 Versorgungsquote der Krankenhäuser nach Ländern 2017

\begin{tabular}{|c|c|c|c|c|c|}
\hline \multirow[t]{2}{*}{ Bundesland } & $\begin{array}{l}\text { Wohnort } \\
\text { des } \\
\text { Patienten }\end{array}$ & $\begin{array}{l}\text { Behandlungs- } \\
\text { ort des } \\
\text { Patienten }\end{array}$ & $\begin{array}{l}\text { Absolute } \\
\text { Differenz }\end{array}$ & $\begin{array}{l}\text { Versorgungs- } \\
\text { quote }\end{array}$ & $\begin{array}{l}\text { Anteil im } \\
\text { eigenen Land } \\
\text { behandelter } \\
\text { Patienten }\end{array}$ \\
\hline & \multicolumn{3}{|c|}{ Anzahl belegter Betten pro Tag ${ }^{1)}$} & \multicolumn{2}{|l|}{ in $\%$} \\
\hline Deutschland & 396.317 & 398.152 & $\mathbf{x}$ & $\mathbf{x}$ & $\mathbf{x}$ \\
\hline Baden-Württemberg & 43.309 & 44.326 & 1.017 & 102,3 & 94,1 \\
\hline Bayern & 58.516 & 60.805 & 2.290 & 103,9 & 96,4 \\
\hline Berlin & 16.249 & 17.986 & 1.737 & 110,7 & 93,6 \\
\hline Brandenburg & 14.280 & 12.638 & -1.642 & 88,5 & 80,0 \\
\hline Bremen & 3.028 & 4.155 & 1.127 & 137,2 & 87,5 \\
\hline Hamburg & 8.032 & 10.968 & 2.936 & 136,5 & 90,3 \\
\hline Hessen & 29.146 & 28.838 & -307 & 98,9 & 89,3 \\
\hline Mecklenburg-Vorpommern & 8.253 & 8.151 & -102 & 98,8 & 92,6 \\
\hline Niedersachsen & 37.029 & 34.503 & -2.526 & 93,2 & 86,0 \\
\hline Nordrhein-Westfalen & 93.450 & 93.206 & -243 & 99,7 & 96,3 \\
\hline Rheinland-Pfalz & 20.236 & 19.242 & -995 & 95,1 & 84,3 \\
\hline Saarland & 5.552 & 5.716 & 164 & 103,0 & 91,1 \\
\hline Sachsen & 20.124 & 20.393 & 269 & 101,3 & 95,3 \\
\hline Sachsen-Anhalt & 12.636 & 11.927 & -710 & 94,4 & 88,9 \\
\hline Schleswig-Holstein & 13.920 & 12.874 & -1.046 & 92,5 & 82,3 \\
\hline Thüringen & 12.560 & 12.426 & -134 & 98,9 & 90,2 \\
\hline \multicolumn{6}{|c|}{$\begin{array}{l}\text { 1) Durchschnittliche vollstationäre Bettenbelegung pro Tag. } \\
\text { Berechnung: Anzahl der Berechnungs-/Belegungstage dividiert durch Anzahl der Kalendertage im Berichtsjahr. } \\
X=\text { Kombination nicht sinnvoll bzw. nicht möglich. }\end{array}$} \\
\hline
\end{tabular}

Krankenhaus-Report 2019

aller Betten. • Tab. 17.5 gibt einen Überblick über ausgewählte Kennzahlen nach Krankenhausgröße und Art des Trägers und zeigt die Veränderungen im Vergleich zum Vorjahr auf.

Die durchschnittliche Bettenauslastung ${ }^{11}$ bezogen auf alle Krankenhäuser lag 2017 bei 77,8 \% (2016: 77,9 \%). Die geringste Bettenauslastung $(61,5 \%)$ hatten Krankenhäuser mit 1 bis 49 Betten aufzuweisen, die höchste (80,5 \%) Einrichtungen

11 Die durchschnittliche Bettenauslastung pro Tag ergibt sich als Quotient aus der Summe der Berechnungs- bzw. Belegungstage im Zähler und der Summe der aufgestellten Betten multipliziert mit der Anzahl der Kalendertage im Berichtsjahr im Nenner. mit 800 und mehr Betten. Allerdings differiert die Bettenauslastung nach Fachabteilungen erheblich ( Abschn. 17.3.2).

Nicht nur bei der Größenstruktur, auch hinsichtlich der Krankenhausträger vollzog sich ein Strukturwandel: Während sich die Anzahl der Krankenhäuser insgesamt von 2007 bis 2017 um 145 (6,9 \%) Einrichtungen verringerte, stieg die Anzahl privater Kliniken um $100(+16,1 \%)$ auf 720 Einrichtungen. Der allgemeine Rückgang der Zahl der Einrichtungen traf folglich die freigemeinnützigen $(16,2 \%)$ und in noch stärkerem Maße die öffentlichen Krankenhäuser (17,3 \%). • Abb. 17.3 zeigt die Auswirkungen dieser Entwicklungen auf die anteilige Verteilung 


\begin{tabular}{|c|c|c|c|c|c|c|c|c|c|c|c|c|}
\hline \multirow{2}{*}{ 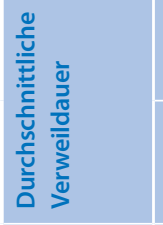 } & 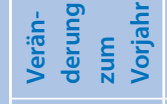 & $\stackrel{\circ}{\stackrel{\circ}{\leftrightarrows}}$ & $\stackrel{n}{p}$ & 1 & 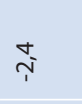 & チ & $\stackrel{\circ}{\grave{r}}$ & $\stackrel{\circ}{=}$ & ̃. & $\underset{T}{\tau}$ & $\Xi$ & n̊ \\
\hline & $\hat{\bar{~}}$ & 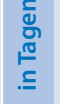 & $\stackrel{n}{n}$ & 1 & $\stackrel{\text { nq }}{N}$ & $\hat{a}$ & $\bar{\infty}$ & 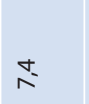 & $\approx$ & $\stackrel{m}{\wedge}$ & $\stackrel{2}{\bumpeq}$ & ầ \\
\hline & 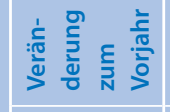 & $\stackrel{\circ}{\subseteq}$ & $\stackrel{\infty}{\rho^{\infty}}$ & 1 & $\stackrel{n}{r}$ & के & $\hat{i}$ & in & గ్ర & $\stackrel{n}{=}$ & $\begin{array}{l}\infty \\
\infty \\
\infty\end{array}$ & $\tilde{\sigma}$ \\
\hline 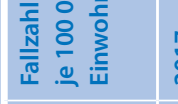 & $\hat{\grave{N}}$ & 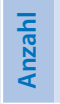 & $\begin{array}{l}\underset{N}{\tilde{N}} \\
\text { in }\end{array}$ & 1 & $\stackrel{\circ}{\sim}$ & $\stackrel{\infty}{\infty}$ & $\stackrel{\circ}{m}$ & 吕 & 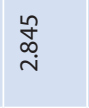 & 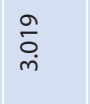 & 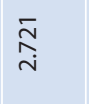 & 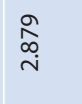 \\
\hline & 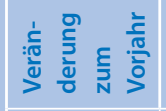 & $\stackrel{\circ}{\leftrightarrows}$ & مै & 1 & $\stackrel{\infty}{\uparrow}$ & 吕 & $\bar{m}$ & $\stackrel{+}{i}$ & 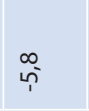 & $\stackrel{a}{=}$ & 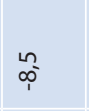 & $\stackrel{0}{\leftarrow}$ \\
\hline 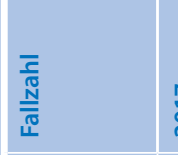 & $\hat{\bar{N}}$ & $\frac{\sqrt{\frac{\pi}{4}}}{\frac{\pi}{\sqrt{5}}}$ & 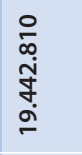 & 1 & $\begin{array}{l}\frac{n}{0} \\
\frac{0}{\alpha} \\
\frac{1}{N}\end{array}$ & 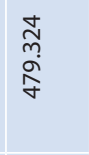 & 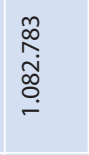 & 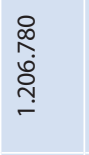 & 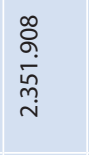 & 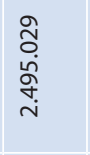 & 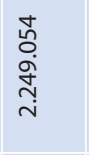 & 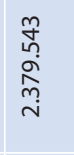 \\
\hline 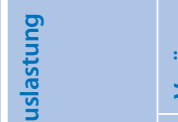 & 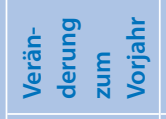 & $\stackrel{\circ}{\stackrel{0}{\leftrightarrows}}$ & $\bar{i}$ & 1 & $\stackrel{t}{\leftarrow}$ & $\stackrel{m}{=}$ & : & ثั & $\stackrel{0}{\circ}$ & $\stackrel{\circ}{\circ}$ & nุ & : \\
\hline 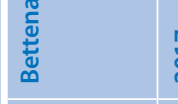 & $\bar{i}$ & 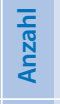 & $\stackrel{\infty}{\stackrel{\infty}{R}}$ & 1 & $\frac{m}{6}$ & $\stackrel{\infty}{\stackrel{\infty}{i}}$ & $\stackrel{\stackrel{2}{ }}{\wedge}$ & $\stackrel{\text { L્n }}{\wedge}$ & $\underset{\substack{t \\
ٌ}}{ }$ & $\stackrel{\mathfrak{L n}^{2}}{\wedge}$ & $\stackrel{\mathbb{2}}{\stackrel{2}{R}}$ & $\stackrel{0}{\infty} \stackrel{\infty}{\wedge}$ \\
\hline$\frac{\bar{d}}{\bar{q}}$ & 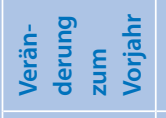 & $\stackrel{\circ}{\subseteq}$ & $\hat{i}$ & 1 & ભે & $\stackrel{\mathfrak{m}_{r}^{n}}{r}$ & $\bar{i}$ & के & ب̣̂ & $=$ & $\bar{i}$ & $\stackrel{\circ}{+}$ \\
\hline 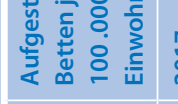 & $\hat{\bar{N}}$ & 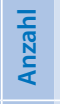 & ชิ & 1 & $a$ & $\bar{\sim}$ & $\hat{m}$ & mे & $\stackrel{n}{\wedge}$ & $\hat{\wedge}$ & 오 & 오 \\
\hline 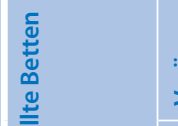 & 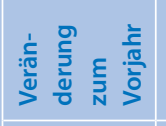 & $\stackrel{\circ}{\subseteq}$ & $\stackrel{m}{i}^{m}$ & 1 & $\stackrel{n_{n}^{n}}{m}$ & $\bar{m}$ & $\stackrel{m}{o}$ & ڤ్ & 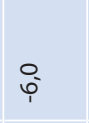 & $\stackrel{\leftrightarrow}{-}$ & $\widehat{\varphi}$ & $\stackrel{d}{a}$ \\
\hline 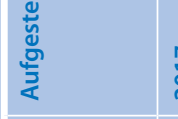 & $\hat{\grave{N}}$ & $\begin{array}{l}\text { 胥 } \\
\frac{N}{<}\end{array}$ & $\frac{\infty}{\stackrel{\infty}{\sigma}}$ & 1 & 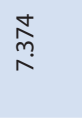 & $\begin{array}{l}\stackrel{n}{0} \\
\stackrel{\leftrightarrow}{-}\end{array}$ & 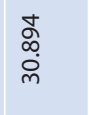 & 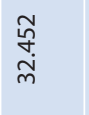 & $\frac{\overline{5}}{8}$ & 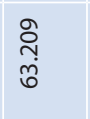 & $\stackrel{b}{i}$ & 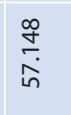 \\
\hline ت & 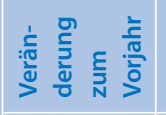 & $\stackrel{\circ}{\leftrightarrows}$ & $\hat{n}$ & $\stackrel{\infty}{\leftarrow}$ & 年 & $\bar{i}$ & $\stackrel{t}{0}$ & $\tilde{o}^{n}$ & $\begin{array}{l}\text { nn } \\
\text { in }\end{array}$ & $=$ & $\begin{array}{l}n \\
n_{0}^{\prime}\end{array}$ & is \\
\hline 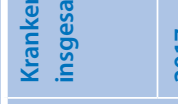 & $\hat{\bar{i}}$ & 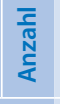 & 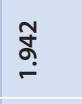 & 乞̊ & 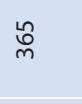 & $\underset{\sim}{\sim}$ & స్ & $\stackrel{\infty}{\infty}$ & $\stackrel{m}{\sim}$ & $\stackrel{\leftrightarrow}{\simeq}$ & શે & 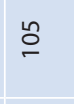 \\
\hline 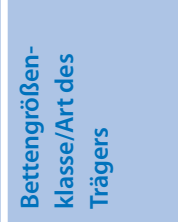 & & & 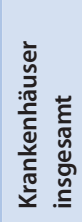 & 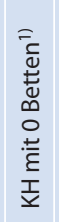 & 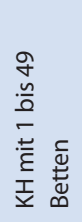 & 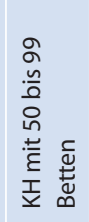 & 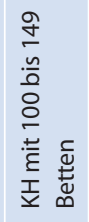 & 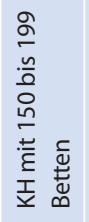 & 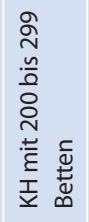 & 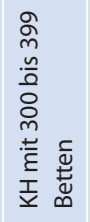 & 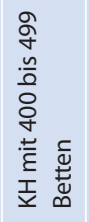 & 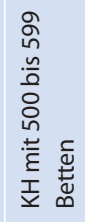 \\
\hline
\end{tabular}




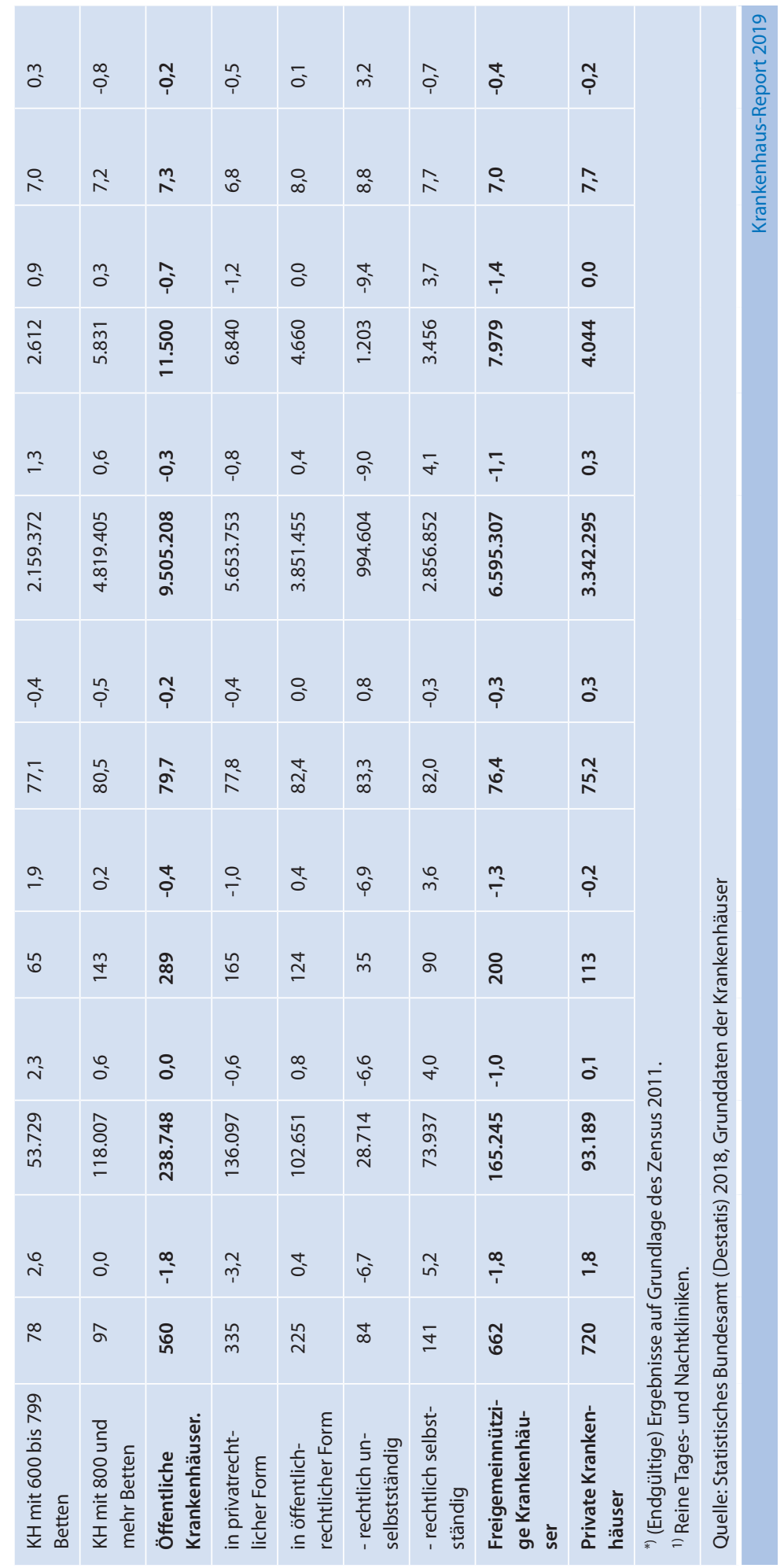




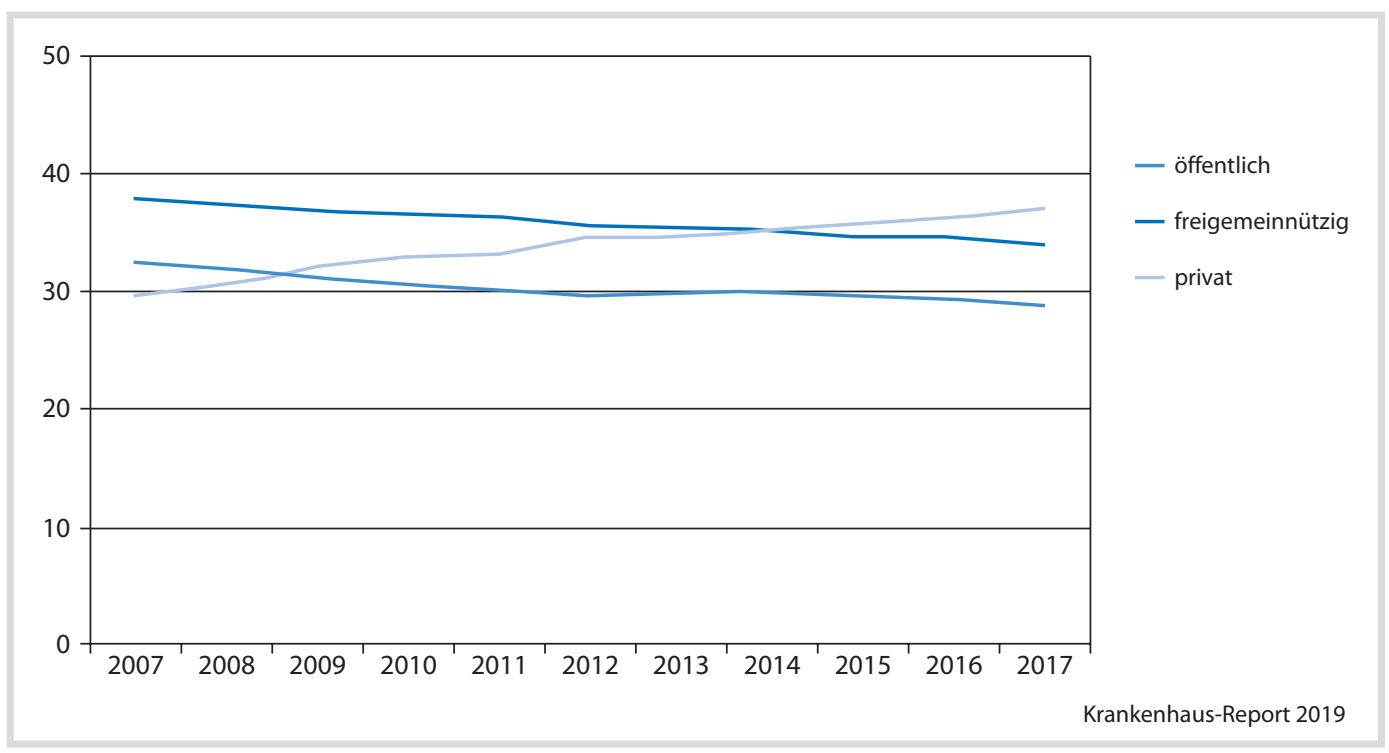

Abb. 17.3 Krankenhäuser nach der Trägerschaft 2007 bis 2017 (Quelle: Statistisches Bundesamt (Destatis) 2018, Grunddaten der Krankenhäuser)

der Krankenhäuser nach Trägern (siehe auch $\mathrm{Zu}$ satztabelle 17.c auf SpringerLink unter https://doi. org/10.1007/978-3-662-58225-1).

Die meisten Krankenhäuser (720 oder 37,1\%) befanden sich 2017 in privater Trägerschaft, gefolgt von den freigemeinnützigen ${ }^{12}$ Krankenhäusern (662 oder 34,1\%) und den öffentlichen Krankenhäusern (560 oder 28,8 \%). Gemessen an der Zahl der verfügbaren Betten dominieren allerdings die öffentlichen Krankenhäuser nach wie vor die Krankenhauslandschaft. Annähernd jedes zweite Bett steht in einem öffentlichen Krankenhaus (238.748 oder $48 \%)$. In freigemeinnütziger Trägerschaft befindet sich jedes dritte Krankenhausbett (165.245 oder 33,2\%) und nur jedes sechste Bett (93.189 oder $18,7 \%)$ steht in einem privaten Krankenhaus. - Abb. 17.4 veranschaulicht die prozentuale Verteilung der Krankenhäuser und der Krankenhausbetten nach Träger- und Rechtsformen im Jahr 2017.

Zwischen Träger und Größenstruktur besteht offenbar ein enger Zusammenhang: Während sich z. B. sehr große Einrichtungen, zu denen in erster Linie die Universitätskliniken gehören, in öffentli-

12 Träger der kirchlichen und freien Wohlfahrtspflege, Kirchengemeinden, Stiftungen oder Vereine. cher Trägerschaft befinden, werden kleine Häuser eher von privaten Trägern betrieben. 2017 verfügte eine Privatklinik über durchschnittlich 129 Betten. Freigemeinnützige Krankenhäuser waren mit 250 Betten annähernd doppelt, öffentliche mit durchschnittlich 426 Betten sogar mehr als dreimal so groß. Allerdings zeigen die Entwicklungen der jüngsten Vergangenheit, dass private Betreiber in den Bereich der Universitätskliniken vorstoßen. ${ }^{13}$ Im Einzelfall sind die rechtlichen Rahmenbedingungen für eine mögliche künftige Privatisierung geschaffen worden ${ }^{14}$ bzw. es werden die

13 Zusammenlegung der Universitätskliniken Gießen und Marburg, Umwandlung in eine $\mathrm{GmbH}$ mit Wirkung vom 2. Januar 2006 und Übernahme von 95 \% der Geschäftsanteile durch die Rhön-Klinikum AG (Hessische Staatskanzlei: Initiativen/Verwaltungsreform/Privatisierung).

14 Landesgesetz über die Errichtung der Universitätsmedizin der Johannes Gutenberg-Universität Mainz (Universitätsmedizingesetz - UMG) vom 10. September 2008 (GVBI. 2008, S. 205), zuletzt geändert durch Artikel 2 des Gesetzes vom 18. August 2015 (GVBI. 2015, S. 196). Das am 1. Januar 2009 in Kraft getretene Gesetz enthält die Option, die rechtsfähige Körperschaft des öffentlichen Rechts in eine Gesellschaft mit beschränkter Haftung (Universitätsmedizin $\mathrm{GmbH}$ ) umzuwandeln - ggf. auch mit Beteiligung privaten Kapitals an dieser $\mathrm{GmbH}$. Einzelheiten zum Formwechsel regelt $§ 25$. 


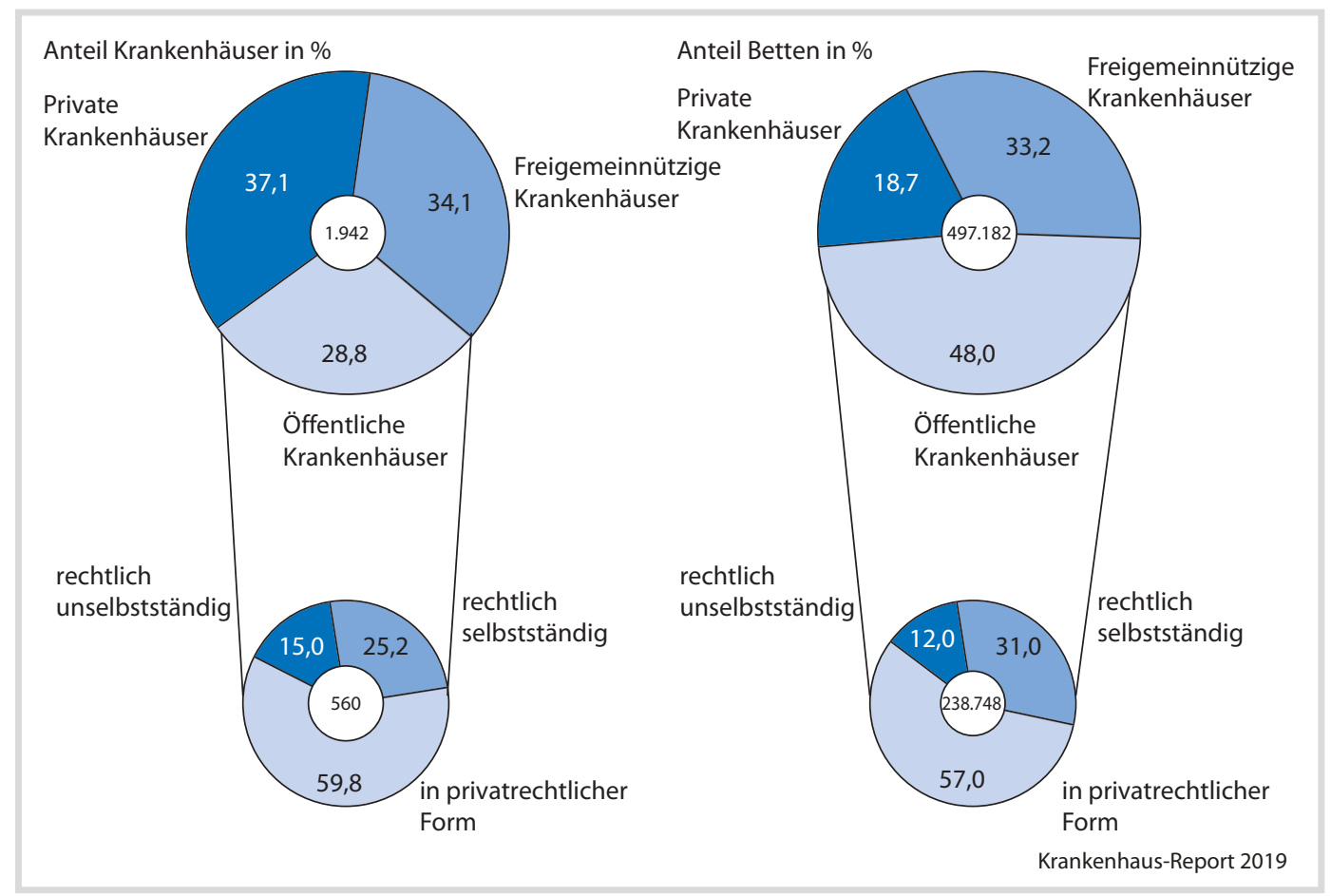

- Abb. 17.4 Trägerstruktur und Rechtsform bei öffentlichen Krankenhäusern 2017 (Quelle: Statistisches Bundesamt (Destatis) 2018, Grunddaten der Krankenhäuser)

rechtlichen Möglichkeiten einer Privatisierung geprüft. ${ }^{15}$

Vor dem Hintergrund veränderter wirtschaftlicher Rahmenbedingungen und der Notwendigkeit zu sparsamer Haushaltsführung haben gestiegene Anforderungen an Wirtschaftlichkeit und Wettbewerbsfähigkeit öffentlicher Einrichtungen dazu geführt, dass immer mehr öffentliche Träger auf diese Veränderungen durch eine rechtliche Verselbstständigung ihrer Einrichtungen reagieren. Seit 2002 wird die Rechtsform öffentlicher Krankenhäuser erfasst; dadurch ist es möglich, den Fortschritt der Überführung öffentlicher Krankenhäuser in eine

15 www.schleswig-holstein.de, Staatskanzlei Schleswig-Holstein: Start > Schwerpunkte > Haushaltskonsolidierung > Die Vorschläge im Detail > Universitätsklinikum Schleswig-Holstein (UKSH). „... Im Bereich von Forschung und Wissenschaft soll nach privaten Investoren für das UKSH gesucht werden. Vor dem Hintergrund der Vereinbarung zwischen dem UKSH, dem Land und den Gewerkschaften werden die rechtlichen Möglichkeiten geprüft und eine materielle Privatisierung des UKSH vorbereitet. ..." privatrechtliche Rechtsform statistisch abzubilden und anhand der Ergebnisse tendenzielle Aussagen über die Entwicklungen in diesem Bereich zu machen.

Mit 335 von insgesamt 560 öffentlichen Krankenhäusern wurden im Jahr 2017 knapp zwei Drittel $(59,8 \%)$ in privatrechtlicher Rechtsform geführt, z. B. als Gesellschaft mit beschränkter Haftung $(\mathrm{GmbH}) ; 2007$ waren es 56,1 \%. Die Zahl der in öffentlich-rechtlicher Form betriebenen öffentlichen Einrichtungen sank auf verbleibende 225 Einrichtungen (40,2 \%). Das entspricht einem Rückgang um 3,7 Prozentpunkte gegenüber 2007. Der Anteil der rechtlich selbstständigen Krankenhäuser, die 2017 als Zweckverband, Anstalt oder Stiftung betrieben wurden, lag bei 25,2\%, der der rechtlich unselbstständigen Einrichtungen (z. B. Regie oder Eigenbetriebe) bei $15 \%$.

Zur sachlichen Ausstattung der Krankenhäuser gehören auch medizinisch-technische Großgeräte und Sondereinrichtungen, wie z. B. Dialysegeräte, Computer- und Kernspin-Tomographen sowie Ko- 


\begin{tabular}{|l|r|r|}
\hline $\begin{array}{l}\text { - Tab. 17.6 Medizinisch-technische Großgeräte und Sondereinrichtungen } 2017 \\
\text { Medizinisch-technisches Großgerät / Sondereinrichtung }\end{array}$ & $\begin{array}{l}\text { 2017 } \\
\text { Anzahl }\end{array}$ & $\begin{array}{l}\text { Veränderung zum } \\
\text { Vorjahr in \% }\end{array}$ \\
\hline Insgesamt & 12.260 & 0,9 \\
\hline Computer-Tomographen & 1.551 & 0,9 \\
\hline Dialysegeräte & 5787 & 1,0 \\
\hline Digitale Subtraktrions-Angiographie-Geräte & 884 & $-1,4$ \\
\hline Gamma-Kameras & 518 & $-3,2$ \\
\hline Herz-Lungen-Maschinen & 502 & 0,4 \\
\hline Kernspin-Tomographen & 1.011 & 2,4 \\
\hline Koronarangiographische Arbeitsplätze & 1.135 & 4,6 \\
\hline Linearbeschleuniger/Kreisbeschleuniger & 400 & $-0,7$ \\
\hline Positronen-Emissions-Computer-Tomographen (PET) & 126 & 0,8 \\
\hline Stoßwellenlithotripter & 324 & $-1,2$ \\
\hline Tele-Kobalt-Therapiegeräte & 22 & 4,8 \\
\hline Quelle: Statistisches Bundesamt (Destatis) 2018, Grunddaten der Krankenhäuser & & \\
\hline
\end{tabular}

ronarangiographische Arbeitsplätze. Insgesamt wurden am 31.12.2017 in den deutschen Krankenhäusern 12.260 medizinisch-technische Großgeräte gezählt. Im Vergleich zum Vorjahr stieg der Bestand um 110 Geräte (0,9 \%). Die höchste Zuwachsrate $(+4,8 \%)$ ist bei den Tele-Kobalt-Therapiegeräten zu verzeichnen, gefolgt von den Koronarangiographischen Arbeitsplätzen (+4,6 \%). Zurückgegangen ist die Zahl der Gammakameras (3,2 \%).

- Tab. 17.6 gibt einen Überblick über Art und Anzahl der in der Krankenhausstatistik erfassten Geräte und Sondereinrichtungen.

\subsubsection{Angebot nach Fachabteilungen}

Fachabteilungen sind organisatorisch abgrenzbare, von Ärztinnen und Ärzten ständig verantwortlich geleitete Abteilungen mit für den jeweiligen Fachbereich typischen Behandlungseinrichtungen. Die Fachabteilungsgliederung orientiert sich an den Gebiets- und Schwerpunktbezeichnungen der Ärzte. Ausgewählte Kennzahlen nach Fachabteilungen für das Jahr 2017 in - Tab. 17.7 vermitteln nicht nur einen Eindruck vom fachlich-medizinischen Versorgungsangebot, sondern zugleich auch vom Behandlungsspektrum der Krankenhäuser.
Allein in den Fachabteilungen Innere Medizin (150 202) und Chirurgie (99 742) waren mehr als die Hälfte aller Krankenhausbetten (50,3 \%) aufgestellt. Hier wurden rund 12 Millionen (61,6\%) aller 19,4 Millionen vollstationären Behandlungsfälle versorgt. Die durchschnittliche Verweildauer in einer allgemeinen Fachabteilung variierte zwischen 2,9 Tagen in der Augenheilkunde und 15,3 Tagen in der Geriatrie. Ausgehend von einer durchschnittlichen Verweildauer von 7,3 Tagen über alle Fachabteilungen dauerte eine Behandlung in der Psychotherapeutischen Medizin/Psychosomatik mit 42,9 Tagen annähernd sechsmal so lange. Sehr unterschiedlich fällt auch der Nutzungsgrad der Betten nach Fachabteilungen aus. Innerhalb der allgemeinen Fachabteilungen reichte er von 45,6 \% in der Nuklearmedizin bis zu 89,3\% in der Geriatrie. In allen psychiatrischen Fachabteilungen (Kinder-/Jugendpsychiatrie und -psychotherapie, Psychiatrie und Psychotherapie sowie Psychotherapeutische Medizin/Psychosomatik) waren die Betten demgegenüber zu $90 \%$ und mehr ausgelastet.

In der Fachabteilung Geriatrie ist das Versorgungsangebot im Vergleich zum Vorjahr am stärksten ausgeweitet worden. Die Anzahl der Fachabteilungen stieg um $12(+3,4 \%)$, die Zahl der verfügbaren Betten um 795 (+4,6\%). Die Zahl der in diesem 
- Tab. 17.7 Ausgewählte Kennzahlen nach Fachabteilungen 2017

\begin{tabular}{|c|c|c|c|c|c|c|}
\hline \multirow{2}{*}{\multicolumn{2}{|c|}{ Fachabteilungsbezeichnung }} & $\begin{array}{l}\text { Fach- } \\
\text { abteilungen } \\
\text { insgesamt }\end{array}$ & $\begin{array}{l}\text { Aufgestellte } \\
\text { Betten }\end{array}$ & $\begin{array}{l}\text { Nutzungs- } \\
\text { grad der } \\
\text { Betten }\end{array}$ & Fallzahl & $\begin{array}{l}\text { Durch- } \\
\text { schnitt- } \\
\text { liche Ver- } \\
\text { weildauer }\end{array}$ \\
\hline & & \multicolumn{2}{|l|}{ Anzahl } & in $\%$ & Anzahl & in Tagen \\
\hline \multicolumn{7}{|c|}{ Fachabteilungen insgesamt } \\
\hline \multirow[t]{20}{*}{-} & \multicolumn{6}{|l|}{ Allgemeine Fachabteilungen } \\
\hline & Augenheilkunde & 286 & 4.416 & 63,5 & 356.483 & 2,9 \\
\hline & Chirurgie & 1.108 & 99.742 & 72,1 & 4.369 .706 & 6,0 \\
\hline & Frauenheilkunde und Geburtshilfe & 801 & 29.055 & 61,0 & 1.730 .737 & 3,7 \\
\hline & Hals-Nasen-Ohrenheilkunde & 635 & 9.418 & 59,5 & 571.660 & 3,6 \\
\hline & Haut- und Geschlechtskrankheiten & 114 & 4.663 & 78,0 & 231.717 & 5,7 \\
\hline & Herzchirurgie & 74 & 5.171 & 80,3 & 138.940 & 10,9 \\
\hline & Innere Medizin & 1.153 & 150.202 & 80,2 & 7.606 .379 & 5,8 \\
\hline & Geriatrie & 360 & 18.121 & 89,3 & 385.120 & 15,3 \\
\hline & Kinderchirurgie & 90 & 1.740 & 63,3 & 126.648 & 3,2 \\
\hline & Kinderheilkunde & 354 & 18.591 & 66,4 & 993.722 & 4,5 \\
\hline & Mund-Kiefer-Gesichtschirurgie & 179 & 2.091 & 65,0 & 114.009 & 4,4 \\
\hline & Neurochirurgie & 184 & 6.988 & 80,2 & 253.252 & 8,1 \\
\hline & Neurologie & 451 & 26.326 & 84,6 & 1.065 .758 & 7,6 \\
\hline & Nuklearmedizin & 100 & 799 & 45,6 & 40.426 & 3,3 \\
\hline & Orthopädie & 409 & 22.204 & 68,5 & 812.668 & 6,8 \\
\hline & Plastische Chirurgie & 139 & 1.969 & 65,4 & 84.977 & 5,5 \\
\hline & Strahlentherapie & 156 & 2.791 & 67,5 & 75.913 & 9,1 \\
\hline & Urologie & 509 & 14.348 & 72,0 & 836.978 & 4,5 \\
\hline & $\begin{array}{l}\text { Sonstige Fachbereiche/ } \\
\text { Allgemeinbetten }\end{array}$ & 206 & 4.603 & 71,6 & 251.030 & 4,8 \\
\hline \multirow[t]{4}{*}{-} & \multicolumn{6}{|l|}{ Psychiatrische Fachabteilungen } \\
\hline & $\begin{array}{l}\text { Kinder-/Jugendpsychiatrie } \\
\text { und -psychotherapie }\end{array}$ & 147 & 6.311 & 91,7 & 61.400 & 34,4 \\
\hline & Psychiatrie und Psychotherapie & 407 & 56.223 & 94,4 & 813.738 & 23,8 \\
\hline & $\begin{array}{l}\text { Psychotherapeutische } \\
\text { Medizin/Psychosomatik }\end{array}$ & 262 & 11.410 & 90,3 & 87.634 & 42,9 \\
\hline
\end{tabular}

Fachbereich behandelten Patientinnen und Patienten nahm gegenüber 2016 um $15.682(+4,2 \%)$ zu. Zuwächse gab es auch im Bereich Neurologie. Vier weitere Fachabteilungen (+0,9\%) mit 629 zusätzlichen Betten (+2,4\%) standen 2017 für die Versorgung der Patientinnen und Patienten zur Verfü- gung, deren Zahl gegenüber 2016 um 4.781 (+0,5\%) stieg. Diesen Entwicklungen steht der Abbau von Überkapazitäten z.B. in den Bereichen Chirurgie und Innere Medizin gegenüber. Trotz einer Verringerung der Zahl der Fachabteilungen Chirurgie um 16 und der verfügbaren Betten um 982 sank die Bet- 
tenauslastung um 0,5 Prozentpunkte auf 72,1\%, weil zugleich 22.732 Patienten weniger behandelt wurden als im Jahr zuvor. In der Inneren Medizin wurde das Versorgungsangebot ebenfalls um 16 Fachabteilungen mit insgesamt 102 Betten verringert. Da jedoch gleichzeitig die Fallzahlen um 40.852 sanken, war in diesem Bereich die Bettenauslastung im Vergleich zum Vorjahr (80,5 \%) um 0,3 Prozentpunkte niedriger. In der Frauenheilkunde und Geburtshilfe wurden 18 Fachabteilungen und 1.073 Betten weniger gezählt als noch im Jahr 2016. Trotz einer um 35.833 geringeren Fallzahl blieb die Bettenauslastung mit 61,0\% annähernd auf Vorjahresniveau (60,8 \%). Noch niedrigere Bettenauslastungen gab es nur in der Hals-Nasen-Ohrenheilkunde $(59,5 \%)$ und der Nuklearmedizin mit $45,6 \%$.

- Abb. 17.2 zeigte bereits deutliche Unterschiede in der Bettendichte nach Bundesländern. Eine genauere Analyse der Unterschiede ermöglicht eine zusätzliche Betrachtung der Bettendichte nach Fachabteilungen. In vierzehn von einundzwanzig ausgewiesenen Fachabteilungen (ohne „Sonstige Fachbereiche/Allgemeinbetten") lag die Bettendichte in Bremen über dem Bundesdurchschnitt, in fünf dieser Fachabteilungen, darunter in der Psychiatrie und Psychotherapie, verfügte Bremen im Vergleich $\mathrm{zu}$ den übrigen Bundesländern über die meisten Betten je 100.000 Einwohner (•Tab. 17.8).

Im Bereich der psychiatrischen Fachabteilungen insgesamt hatte Schleswig-Holstein 2017 die höchste Bettendichte. Während im Bundesdurchschnitt 90 Betten je 100.000 Einwohner in einer psychiatrischen Fachabteilung zur Verfügung standen, waren es in Schleswig-Holstein 105 Betten je 100.000 Einwohner. Demgegenüber gab es in Berlin lediglich 73 Betten je 100.000 Einwohner in einer psychiatrischen Fachabteilung. Im Fachbereich Plastische Chirurgie gibt es nicht in allen Bundesländern ein stationäres Versorgungsangebot.

\subsubsection{Personal der Krankenhäuser}

Am 31.12.2017 wurden gut 1,24 Millionen Beschäftigte in den Krankenhäusern gezählt, 22.065 Personen bzw. 1,8 \% mehr als am 31.12.2016. 186.021 Beschäftigte waren als hauptamtliche Ärzte und
Ärztinnen tätig; gut eine Million Beschäftigte (darunter 84.186 Schüler/-innen und Auszubildende) waren dem nichtärztlichen Dienst zuzurechnen. Im Vergleich zum Vorjahr stieg die Zahl der hauptamtlichen Ärzte und Ärztinnen um 5.649 (+3,1 \%) Beschäftigte, die Zahl der im nichtärztlichen Dienst tätigen Krankenhausmitarbeiter und -mitarbeiterinnen nahm um 16.416 (+1,6\%) Beschäftigte zu. $25,1 \%$ des ärztlichen und 48,3 \% des nichtärztlichen Personals sind teilzeit- oder geringfügig beschäftigt. Um den Auswirkungen unterschiedlicher Beschäftigungsmodelle (Vollzeit, Teilzeit oder geringfügige Beschäftigung sowie kurzfristige Beschäftigung) angemessen Rechnung zu tragen, wird zusätzlich zur Zahl der Beschäftigten am Erhebungsstichtag 31. Dezember des Jahres die Anzahl der Vollkräfte im Jahresdurchschnitt ${ }^{16}$ (Vollzeitäquivalente) erhoben. Die Gesamtzahl der Vollkräfte erhöhte sich gegenüber 2016 um 13.881 bzw. 1,6 \% auf 894.400 Vollkräfte, von denen 161.208 (18 \%) im ärztlichen Dienst und 733.193 (82 \%) im nichtärztlichen Dienst arbeiteten; 328.327 nichtärztliche Vollkräfte wurden allein im Pflegedienst gezählt.

Die Krankenhausstatistik liefert zudem Informationen über das Geschlecht und den Beschäftigungsumfang der Beschäftigten. 46,3 \% der hauptamtlichen Ärzte waren im Jahr 2017 Frauen (•Tab. 17.9). Damit entspricht der Frauenanteil annähernd dem Vorjahresniveau (46,5\%); gegenüber 2007 stieg der Anteil um 6,0 Prozentpunkte. Mit steigender Hierarchiestufe nimmt der Frauenanteil an den Krankenhausärzten deutlich ab. Während zu Beginn der ärztlichen Laufbahn gut die Hälfte aller Assistenzarztstellen (55,9\%) von Frauen besetzt wurde, war es bei den Oberärzten noch knapp ein Drittel $(31,5 \%)$ der Stellen. Der Frauenanteil an den leitenden Ärzten lag bei nur noch 12,5\%.

Deutlich verändert hat sich in den vergangenen zehn Jahren auch der Beschäftigungsumfang. 2007 war jede vierte hauptamtliche Ärztin (25,7\%) teilzeit- oder geringfügig beschäftigt; 2017 war es bereits jede Dritte (36,5\%). Bei ihren männlichen Kollegen verdreifachte sich im gleichen Zeitraum

16 Zur Ermittlung der Vollkräfte im Jahresdurchschnitt werden die unterschiedlichen Beschäftigungsmodelle auf die volle jährliche tarifliche Arbeitszeit umgerechnet. Überstunden und Bereitschaftsdienste werden nicht in die Berechnung einbezogen. 


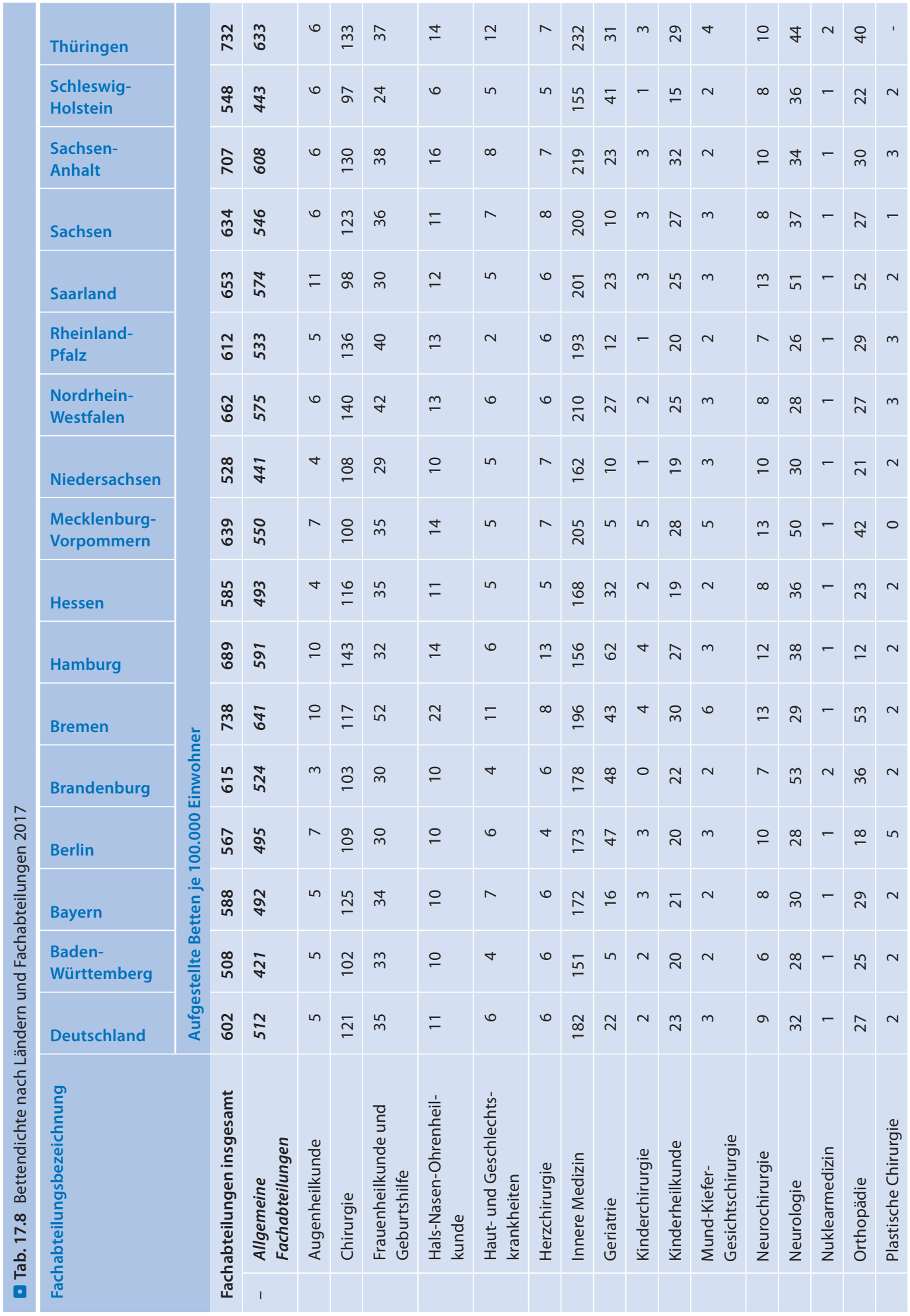




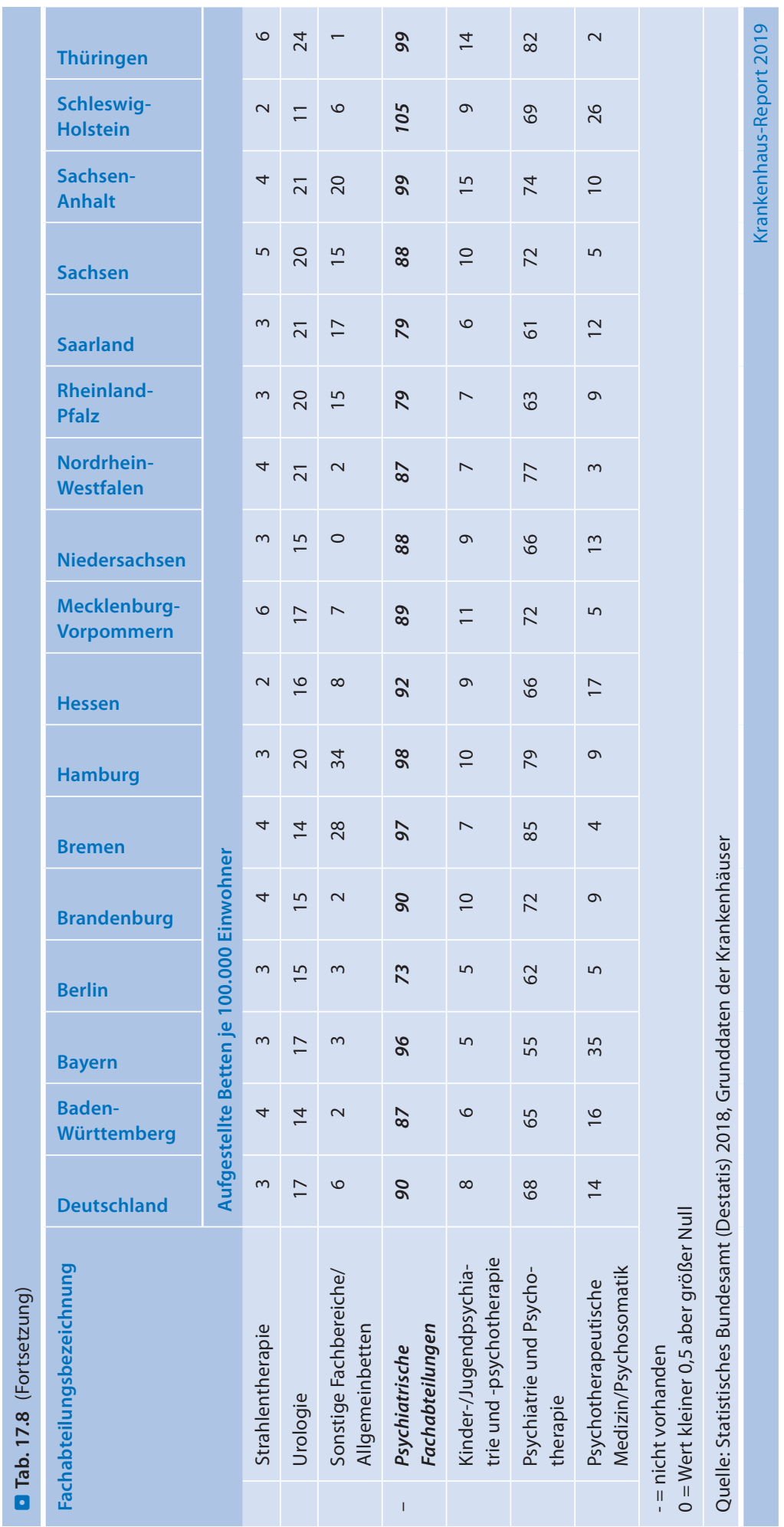




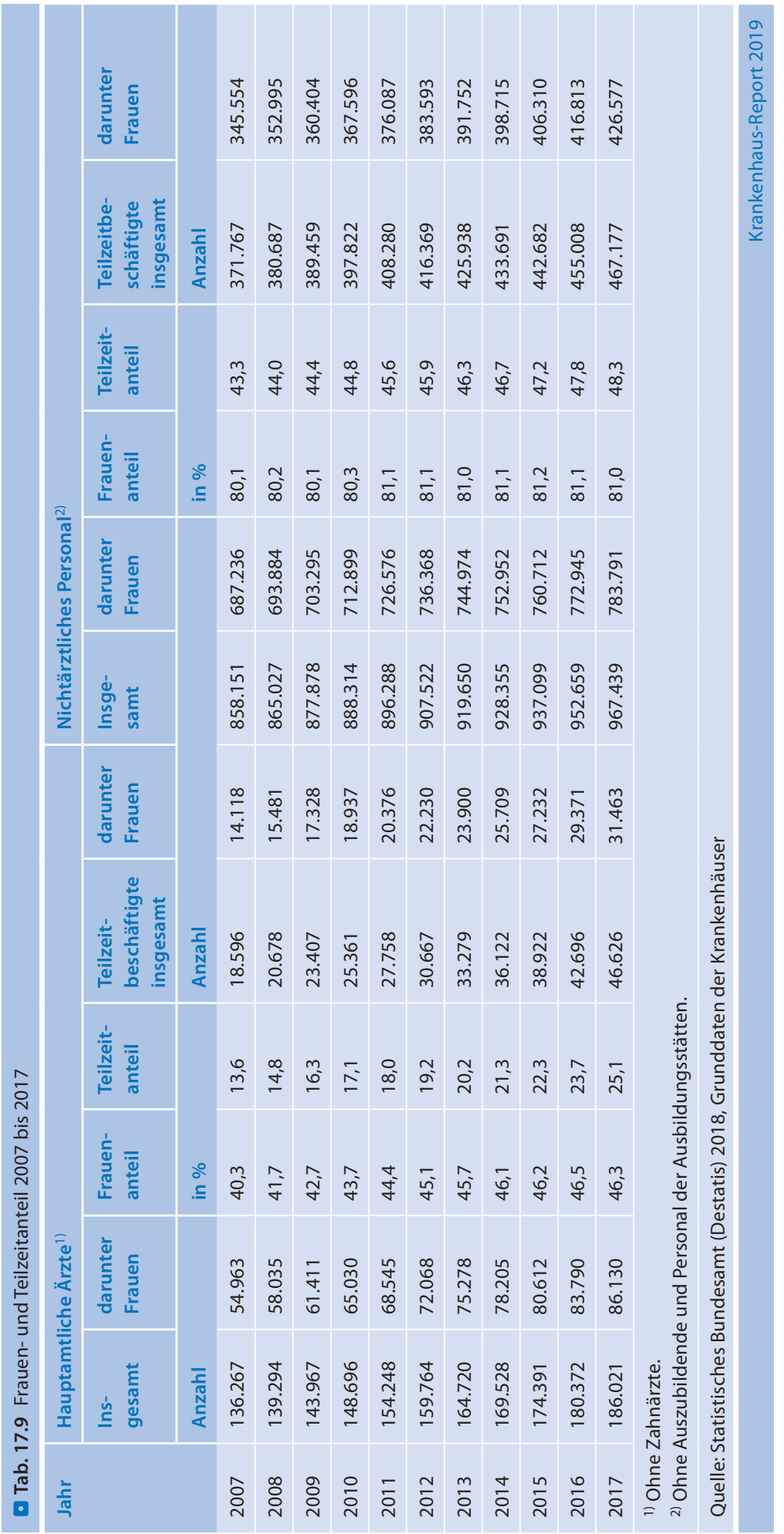


annähernd der Anteil der teilzeit- oder geringfügig Beschäftigten von 5,5\% auf 15,2\%. Insgesamt gab es 46.626 (25,1 \%) hauptamtliche Ärzte und Ärztinnen, die 2017 in einem Teilzeitarbeitsverhältnis standen oder geringfügig beschäftigt waren.

Mit 967.439 Beschäftigten (ohne Schüler/Schülerinnen und Auszubildende) war die Zahl der im nichtärztlichen Dienst tätigen Krankenhausmitarbeiter gut fünfmal so hoch wie die der Beschäftigten im ärztlichen Dienst. Die mit Abstand meisten nichtärztlichen Beschäftigten (437.648) waren im Pflegedienst tätig (45,2\%). An zweiter Stelle folgten der medizinisch-technische Dienst (z. B. Laboratoriums- und Radiologieassistentinnen und -assistenten, Krankengymnastinnen und -gymnasten) mit 20,5 \% und der Funktionsdienst (z. B. Personal im Operationsdienst, in der Ambulanz und in Polikliniken) mit 14,5\%.

Der Frauenanteil beim nichtärztlichen Personal lag mit 81,0\% deutlich über dem Anteil weiblicher Beschäftigter beim ärztlichen Personal (46,3\%). Während Frauen vorwiegend im Pflegedienst beschäftigt waren $(85,0 \%)$, dominierten beim Personal des technischen Dienstes und des Krankentransportdienstes Männer mit 91,2\% und 78,1\%. Der Anteil teilzeit- und geringfügig Beschäftigter ist im nichtärztlichen Bereich im Vergleich zu den hauptamtlichen Ärzten und Ärztinnen annähernd zweimal so hoch: 48,3 \% im Jahr 2017. Zehn Jahre zuvor waren es gerade mal 43,3\%.
Zusammenfassend gibt • Abb. 17.5 einen Überblick über die Personalstruktur der Krankenhäuser auf der Grundlage der für 2017 ermittelten 894.400 Vollkräfte nach Beschäftigtengruppen.

Die Personalstruktur variierte je nach Krankenhausträger. Bei den Krankenhäusern privater Träger gehörten $18,7 \%$ aller Vollkräfte dem ärztlichen Personal an, bei den freigemeinnützigen Krankenhäusern waren dies lediglich 17,7 \%. Der Anteil der im Pflegedienst tätigen Vollkräfte ist am höchsten bei den freigemeinnützigen und den privaten Krankenhäusern mit jeweils 39,4\% und am niedrigsten bei den öffentlichen Krankenhäusern mit 34,5 \% (siehe auch Zusatztabelle 17.d auf SpringerLink unter https://doi.org/10.1007/978-3-662-58225-1).

Seit 2009 wird zusätzlich zu den Vollkräften mit direktem Beschäftigungsverhältnis beim Krankenhaus die Zahl der Vollkräfte ohne direktes Beschäftigungsverhältnis beim Krankenhaus erhoben. Im Jahr 2017 handelte es sich hierbei um 23.038 Vollkräfte, davon 2.704 im ärztlichen Dienst und 20.334 im nichtärztlichen Dienst Beschäftigte, die z. B. im Personal-Leasing-Verfahren eingesetzt wurden. Entscheidend ist, dass die Leistung vom Krankenhaus erbracht wird ${ }^{17}$ und dazu das Personal etwa

17 Personal einer Fremdfirma, die z. B. die Reinigung übernommen hat, wird nicht erfasst; hier gehört die („outgesourcte") Reinigung nicht mehr zu den Leistungen des Krankenhauses.

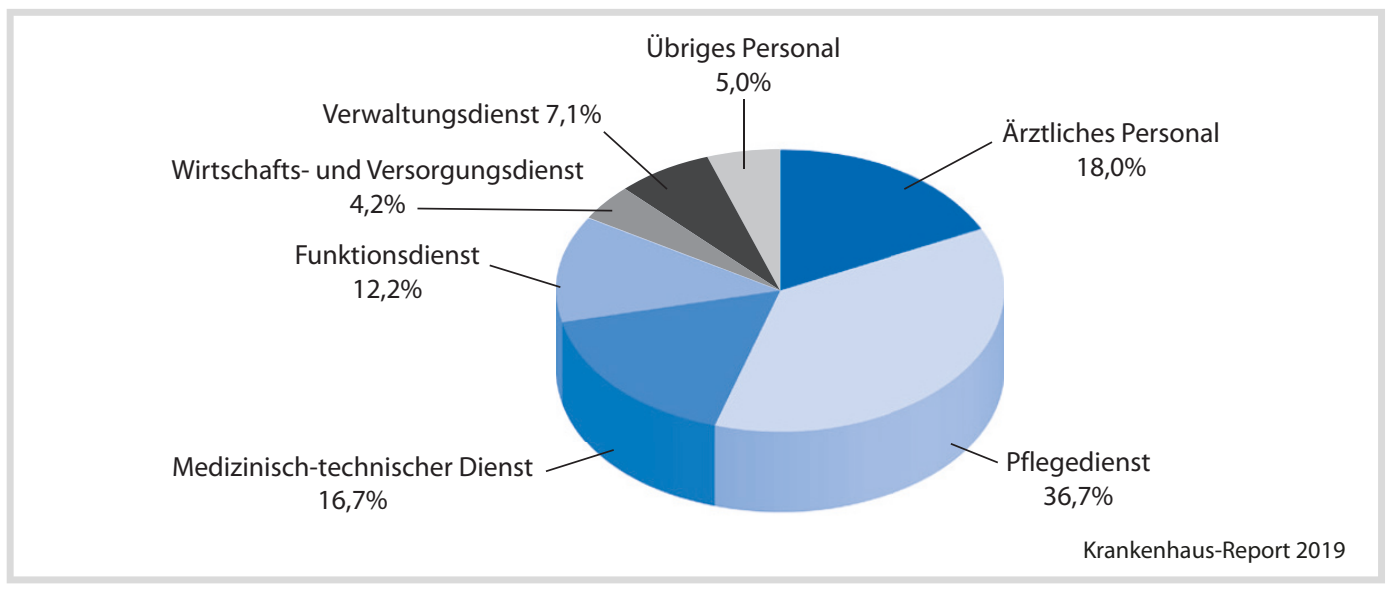

- Abb. 17.5 Personalstruktur der Krankenhäuser 2017 (Vollkräfte) (Quelle: Statistisches Bundesamt (Destatis) 2018, Grunddaten der Krankenhäuser) 
durch Zeitarbeitnehmer und -arbeitnehmerinnen verstärkt wird. Beim ärztlichen Personal ohne direktes Beschäftigungsverhältnis kann es sich um Honorarkräfte oder um Ärzte und Ärztinnen handeln, die über (konzerninterne) Personalgesellschaften im Krankenhaus eingesetzt werden. Beim nichtärztlichen Personal ohne direktes Beschäftigungsverhältnis spielen sowohl konzerninterne Personalgesellschaften als auch Zeitarbeit eine Rolle.

\subsection{Die Inanspruchnahme von Krankenhausleistungen}

Das vielfältige Spektrum der Behandlungsformen im Krankenhaus geht weit über die klassische vollstationäre, d. h. ganztägige Behandlung hinaus und umfasst auch teil-, vor- und nachstationär sowie ambulant erbrachte Leistungen. Diese ineinandergreifenden Behandlungsformen werden in der Krankenhausstatistik in unterschiedlicher Tiefe abgebildet, wobei der herkömmlichen vollstationären Behandlung das Hauptinteresse gilt.

\subsubsection{Vollstationäre Behandlungen}

19,4 Millionen vollstationär behandelte Patienten ${ }^{18}$ wurden im Berichtsjahr 2017 gezählt. Erstmals seit dem Jahr 2005 (16,5 Millionen Fälle) war im Vergleich zum Vorjahr (2016: 19,5 Millionen Fälle) ein Rückgang um rund 90.000 vollstationär behandelte Patienten oder 0,5\% zu verzeichnen.

Die Summe der 2017 erbrachten vollstationären Berechnungs- und Belegungstage ${ }^{19}$ sank gegenüber

18 Die Fallzahl in den Grunddaten der Krankenhäuser ermittelt sich aus der Summe der vollstationären Aufnahmen (Patientenzugang) und der Summe der Entlassungen aus vollstationärer Behandlung einschließlich der Sterbefälle (Patientenabgang) im Berichtsjahr, dividiert durch 2.

19 Berechnungstage sind die Tage, für die tagesgleiche Pflegesätze (Basispflegesatz, Abteilungspflegesatz oder teilstationäre Pflegesätze) in Rechnung gestellt (berechnet) werden. Unter einem Belegungstag wird ein Tag verstanden, an dem ein aufgestelltes Bett von einem Patienten bzw. einer Patientin vollstationär belegt wurde. Innerhalb des pauschalierten Entgeltsystems ist der Belegungstag das Äquivalent zum Begriff des Berechnungstags innerhalb der Bundespflegesatzverordnung.
2016 um gut 1 Million oder 0,7 \%. Ein Krankenhausaufenthalt dauerte im Jahr 2017 wie im Vorjahr durchschnittlich 7,3 Tage $^{20}$. Gegenüber 2007 (8,3 Tage) ist die Dauer des Krankenhausaufenthaltes um einen Tag zurückgegangen.

\subsubsection{Teil-, vor- und nachstationäre Behandlungen}

Um der zunehmenden Bedeutung von nicht rein vollstationären Behandlungsformen in Krankenhäusern gerecht zu werden, werden seit 2002 neben den vollstationären Behandlungen auch einzelne Merkmale im Bereich der teil-, vor- und nachstationären Behandlungen in der Krankenhausstatistik detaillierter erfasst. ${ }^{21}$

Unter einer teilstationären Behandlung versteht man eine Krankenhausleistung, die eine regelmäßige Verweildauer im Krankenhaus von weniger als 24 Stunden erfordert. Sie wird vorwiegend in einer von insgesamt 65 reinen Tages oder Nachtkliniken angeboten. Die Patientinnen und Patienten verbringen dabei nur den entsprechenden Tagesabschnitt mit der ärztlichen Behandlung, die restliche Zeit aber außerhalb des Krankenhauses. 2017 wurden in den Krankenhäusern gut 790.900 teilstationäre Behandlungen ${ }^{22}$ durchgeführt, 2,2 \% mehr als im Jahr zuvor. Knapp die Hälfte (43,0 \%) aller teilstationä-

20 Die durchschnittliche Verweildauer ergibt sich als Quotient aus der Summe der Berechnungs- bzw. Belegungstage und der Fallzahl.

21 Vor Inkrafttreten der Ersten Novellierung der KHStatV wurde lediglich die Anzahl der aus teilstationärer Behandlung entlassenen Patientinnen und Patienten erhoben.

22 Die Fallzählung (Anzahl der Behandlungen) hängt von der Art der Abrechnung teilstationärer Leistungen ab: Sind für teilstationäre Leistungen, die über Entgelte nach $\S 6$ Abs. 1 KHEntgG (Krankenhausentgeltgesetz) abgerechnet werden, fallbezogene Entgelte vereinbart worden, zählt jede abgerechnete Patientin/jeder abgerechnete Patient als ein Fall; sind dagegen tagesbezogene Entgelte vereinbart worden, werden Patientinnen und Patienten, die wegen derselben Erkrankung mehrfach teilstationär behandelt wurden, je Quartal als ein Fall gezählt. Die Quartalszählung ist auch anzuwenden bei teilstationären Leistungen nach $\S 13$ Abs. 1 BPfIV (Bundespflegesatzverordnung), die mit einem gesonderten Pflegesatz abgerechnet werden. 
ren Behandlungen fand in der Inneren Medizin statt. Innerhalb dieses Fachbereichs entfielen allein 42,2 \% aller Behandlungen auf das Teilgebiet Nephrologie (z. B. Dialyse), weitere $15,6 \%$ auf das Teilgebiet Hämatologie und internistische Onkologie (z. B. Chemotherapie).

Vorstationäre Behandlungen werden im Vorfeld einer anstehenden vollstationären Behandlung erbracht, z. B. für Voruntersuchungen. In diesem Bereich wurden knapp 4,7 Millionen Behandlungsfälle im Jahr 2017 gezählt, rund 14.400 bzw. 0,3\% mehr als 2016. Im Vergleich zu 2005 ist die Zahl der vorstationären Behandlungen mit einem Plus von $138,4 \%$ mit Abstand am deutlichsten gestiegen. Jede dritte Behandlung dieser Art (34,4\%) wurde 2017 in der Fachabteilung Chirurgie durchgeführt, gefolgt von der Inneren Medizin mit 22,1 \% aller vorstationären Behandlungen.
Nachstationäre Behandlungen finden im Anschluss an einen vollstationären Krankenhausaufenthalt statt. Ihre Zahl lag im Jahr 2017 bei rund 1,1 Millionen Behandlungen. Das waren im Vergleich zum Vorjahr 0,4\% weniger. Die meisten nachstationären Behandlungen erfolgten in der Chirurgie (37,1\%), weitere $13,4 \%$ in der Inneren Medizin.

Zusammengenommen erweiterten die genannten Behandlungsformen das Leistungsvolumen der Krankenhäuser im Jahr 2017 um rund 6,5 Millionen Behandlungsfälle.

\subsubsection{Ambulante Operationen}

Seit 2002 wird in der Krankenhausstatistik darüber hinaus auch die Anzahl der ambulanten Operatio-

- Tab. 17.10 Behandlungsformen in Krankenhäusern

\begin{tabular}{|c|c|c|c|c|c|}
\hline \multirow[t]{3}{*}{ Jahr } & \multicolumn{4}{|c|}{ Behandlungsfälle ${ }^{1)}$} & \multirow{2}{*}{$\begin{array}{l}\text { Ambulante } \\
\text { Operationen }\end{array}$} \\
\hline & vollstationär & teilstationär & vorstationär & nachstationär & \\
\hline & \multicolumn{5}{|l|}{ Anzahl } \\
\hline 2005 & 16.539 .398 & 527.213 & 1.965 .027 & 654.277 & 1.371 .708 \\
\hline 2006 & 16.832 .883 & 623.657 & 2.266 .670 & 703.488 & 1.513 .716 \\
\hline 2007 & 17.178 .573 & 675.082 & 2.714 .169 & 781.197 & 1.638 .911 \\
\hline 2008 & 17.519 .579 & 702.649 & 2.991 .986 & 820.371 & 1.758 .305 \\
\hline 2009 & 17.817 .180 & 667.093 & 3.298 .544 & 875.259 & 1.813 .727 \\
\hline 2010 & 18.032 .903 & 673.080 & 3.510 .861 & 905.602 & 1.854 .125 \\
\hline 2011 & 18.344 .156 & 686.364 & 3.820 .969 & 958.163 & 1.865 .319 \\
\hline 2012 & 18.620 .442 & 734.263 & 4.092 .333 & 988.307 & 1.867 .934 \\
\hline 2013 & 18.787 .168 & 724.685 & 4.336 .205 & 993.593 & 1.897 .483 \\
\hline 2014 & 19.148 .626 & 743.561 & 4.581 .160 & 1.031 .277 & 1.953 .727 \\
\hline 2015 & 19.239 .574 & 764.745 & 4.656 .886 & 1.057 .015 & 1.978 .783 \\
\hline 2016 & 19.532.779 & 773.807 & 4.670 .177 & 1.075 .006 & 1.962 .051 \\
\hline 2017 & 19.442 .810 & 790.947 & 4.684 .575 & 1.070 .750 & 1.970 .516 \\
\hline Vergleichsjahr & \multicolumn{5}{|c|}{ Veränderung in \% } \\
\hline 2016 & $-0,5$ & 2,2 & 0,3 & $-0,4$ & 0,4 \\
\hline 2005 & 17,6 & 50,0 & 138,4 & 63,7 & 43,7 \\
\hline \multicolumn{6}{|c|}{$\begin{array}{l}\text { 1) Vor Inkrafttreten der 1. Novellierung der KHStatV wurde lediglich die Anzahl der aus teilstationärer Behandlung ent- } \\
\text { lassenen Patientinnen und Patienten erhoben. }\end{array}$} \\
\hline
\end{tabular}


nen im Krankenhaus erfasst. Nach $₫ 115 b$ Fünftes Buch Sozialgesetzbuch (SGB V) sind Krankenhäuser zur Durchführung ambulanter Operationen zugelassen, und zwar in dem Umfang, der in einem vom Spitzenverband Bund der Krankenkassen, der Deutschen Krankenhausgesellschaft oder den Bundesverbänden der Krankenhausträger gemeinsam und der Kassenärztlichen Bundesvereinigung vereinbarten Katalog ambulant durchführbarer Operationen und sonstiger stationsersetzender Eingriffe festgelegt ist.

Knapp zwei Drittel aller Krankenhäuser (60,5\%) führten im Jahr 2017 knapp 2 Millionen ambulante Operationen durch. Im Vergleich $\mathrm{zu}$ 2005 ist die Zahl der ambulanten Operationen auf das Eineinhalbfache gestiegen. Dabei entwickelte sich dieser Leistungsbereich mit anfänglichen jährlichen Steigerungsraten von bis zu 60 \% äußerst dynamisch. Seit 2007 blieben die jährlichen Steigerungsraten unter $10 \%$; im Vergleich zu 2016 stieg die Zahl ambulanter Operationen nur noch um 0,4\%. Das Potential der Krankenhäuser auf dem Gebiet ambulanter Gesundheitsversorgung scheint ausgeschöpft (•Tab. 17.10).

Open Access Dieses Kapitel wird unter der Creative Commons Namensnennung 4.0 International Lizenz(http://creativecommons. org/licenses/by/4.0/deed.de) veröffentlicht, welche die Nutzung, Vervielfältigung, Bearbeitung, Verbreitung und Wiedergabe in jeglichem Medium und Format erlaubt, sofern Sie den/die ursprünglichen Autor(en) und die Quelle ordnungsgemäß nennen, einen Link zur Creative Commons Lizenz beifügen und angeben, ob Änderungen vorgenommen wurden.

Die in diesem Kapitel enthaltenen Bilder und sonstiges Drittmaterial unterliegen ebenfalls der genannten Creative Commons Lizenz, sofern sich aus der Abbildungslegende nichts anderes ergibt. Sofern das betreffende Material nicht unter der genannten Creative Commons Lizenz steht und die betreffende Handlung nicht nach gesetzlichen Vorschriften erlaubt ist, ist für die oben aufgeführten Weiterverwendungen des Materials die Einwilligung des jeweiligen Rechteinhabers einzuholen. 